\title{
REVISION OF OCHROSIA (APOCYNACEAE) IN MALESIA
}

\author{
HENDRIAN \\ Bogor Botanic Garden, Indonesian Institute of Sciences, \\ Jalan Ir. H. Juanda 13, Bogor, Indonesia
}

\section{SUMMARY}

The genus Ochrosia Juss. is revised in the Malesian region. Thirteen species are recognized as Ochrosia, including one new species, $O$. basistamina. Seven species previously placed in Neisosperma Raf. are now included in Ochrosia.

The names Ochrosia section Echinocaryon F. Muell. and Ochrosia section Ochrosia are applied for the fibrous-fruited species and cavity-fruited species of Ochrosia, respectively. Neisosperma Raf. is reduced to Ochrosia section Echinocaryon F. Muell.

Separate keys are given for the species of both sections.

Key words: Neisosperma, Ochrosia, Ochrosia section Echinocaryon, Ochrosia section Ochrosia, Malesia, taxonomic revision.

\section{INTRODUCTION}

\section{The history}

In the most recent classification of the Apocynaceae by Endress \& Bruyns (2000) the genus Ochrosia is placed in the tribe Vinceae of the subfamily Rauvolfioideae. The tribe is characterized mainly by the possession of unadorned seeds, a basal-collared style head and corolla lobes which are usually sinistrorsely contorted, except in Kopsia, Ochrosia, and Neisosperma.

Ochrosia was proposed by De Jussieu in his Genera Plantarum (1789), without any description or type indicated, on the 'bois jaune' collected by Commerson in Bourbon (= Réunion). This plant was preserved in P (Herb. Jussieu no. 7170) (Boiteau et al., 1974). It was given a specific name in 1791 by Gmelin: Ochrosia borbonica, which is a nomen nudum. This epithet was antedated by $O$. maculata Jacq. (1791), also based on a specimen collected from Bourbon (Markgraf, 1979).

Delimitation of the genus was soon found to be problematic. As more species were discovered botanists became aware of fruit differences within the genus. It was Mueller (1871) who first used this character to discern two sections: Ochrosia section Lactaria with a massive, thick endocarp containing two lateral cavities filled with spongy tissue, and Ochrosia section Echinocaryon with an endocarp split into diverging fibres.

This classification was followed by Valeton (1895) (at subgeneric level) who put O. elliptica Labill., O. calocarpa (Hassk.) Miq. (= O. elliptica Labill.), O. ackeringae (Teijsm. \& Binn.) Miq., O. coccinea (Teijsm. \& Binn.) Miq., O. moorei (F. Muell.) F. Muell., and $O$. sandwichensis Hillebr. $(O$. sandwichensis var. $\beta$ Hillebr. $=O$. compta K. Schum.) (which are all cavity-fruited species) under Ochrosia subgenus Lactaria F. Muell., and O. salubris Blume (= O. oppositifolia (Lam.) K. Schum.), O. kilneri 

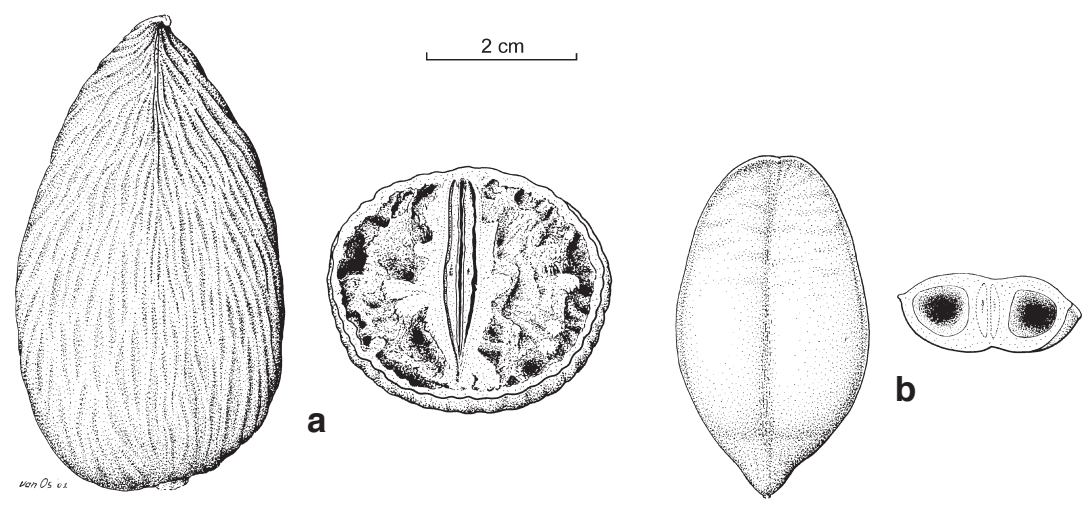

b

Fig. 1. Two fruit types of Ochrosia. a. Fibrous-fruited type; b. cavity-fruited type.

F. Muell., O.acuminata Trimen ex Valeton, and O.glomerata (Blume) F. Muell. (which are all fibrous-fruited species (Fig. 1)) under Ochrosia subgenus Echinocaryon F. Muell. But he incorrectly placed $O$. borbonica J.F. Gmel. (which is a cavity-fruited species (Fig. 1)) into Ochrosia subgenus Echinocaryon F. Muell.

At the generic level, the cavity-fruited species have been placed in the genus Bleekeria Hassk. and in Excavatia Markgr. Hasskarl (1855) proposed Bleekeria with 2 species, B. kalokarpa Hassk. and B. salubris Hassk. The concept of this genus was rather confusing. The generic description applied to the cavity-fruited species but also included Bleekeria salubris Hassk. (= O. oppositifolia (Lam.) K. Schum.) which is a fibrousfruited species. In 1923 the genus was emended by Koidzumi who took out Bleekeria salubris and moved it into Lactaria Rumph. ex Raf. He then added some other cavityfruited species into Bleekeria. In 1927 Markgraf proposed Excavatia with 3 cavityfruited species: E. litoralis (Merr.) Markgr. (= O. ackeringae (Teijsm. \& Binn.) Miq., E. coccinea (Teijsm. \& Binn.) Markgr. (= O. coccinea (Teijsm. \& Binn.) Miq.) and E. mariannensis (A.DC.) Markgr. (=O. mariannensis A.DC.).

Conversely Pichon (1947) kept the cavity-fruited species as the true Ochrosia Juss. This was supported by Markgraf (1979) who mentioned “adult fruits of $O$. borbonica - the type species of Ochrosia Juss. - contain these holes, though only in the lower half or third". However, Pichon treated the whole complex as one genus, and subdivided it into three sections: Ochrosia section Lactaria F. Muell., Ochrosia section Echinocaryon F. Muell., and Ochrosia section Phragmochrosia Pichon. Section Phragmochrosia was proposed based on a single species - Ochrosia apoensis, and characterized by having both numerous fibres and rudimentary lateral cavities. Unfortunately, except for the fibrous endocarps I do not see the cavities mentioned by Pichon. Therefore, I prefer to reduce Ochrosia section Phragmochrosia and place it in Ochrosia section Echinocaryon.

Fosberg \& Sachet (1977) agreed with Pichon who kept the cavity-fruited species as the true Ochrosia Juss., and thought that it was necessary to restrict the name Ochrosia Juss. to the portion containing the type (the cavity-fruited species) and tried to find a name for the other portion. They did not agree with Boiteau et al. (1974) who had proposed Calpicarpum G. Don emend. Boiteau for the species with 
loosely fibrous endocarps, and instead resurrected the name Neisosperma Raf. Fosberg et al. (1977) placed Bleekeria Hassk., Ochrosia section Lactaria F. Muell., Ochrosion St.-Lag. (nomen nudum), and Excavatia Markgr. in synonymy of Ochrosia Juss., and Lactaria Rumph. ex Raf., Pseudochrosia Blume, Ochrosia section Echinocaryon F. Muell. and Calpicarpum G. Don emend. Boiteau as synonyms of Neisosperma Raf. This application was then followed by Markgraf (1979), Boiteau \& Allorge (1981), and Forster (1993) who used Neisosperma Raf. for the fibrous-fruited species and Ochrosia Juss. for the cavity-fruited species.

In contrast Leeuwenberg (1987) put cavity-fruited and fibrous-fruited species together into one genus, reducing Calpicarpum G. Don emend. Boiteau, Neisosperma Raf., Lactaria Rumph. ex Raf., Pseudochrosia Blume, Bleekeria Hassk., and Excavatia Markgr. to the synonymy of Ochrosia Juss.

\section{Ochrosia-Neisosperma}

In proposing the segregation of Neisosperma from Ochrosia, Fosberg \& Sachet $(1972,1974)$ justified recognition of the 2 genera based on differences in fruit (which is discussed above) and inflorescence characters (in those two publications they incorrectly spelled the genus as Neiosperma). However, as the inflorescences of very few species were studied in detail (the concept of the Neisosperma inflorescence was largely based on the study of living material of N. oppositifolia (Lam.) Fosberg \& Sachet (= Ochrosia oppositifolia (Lam.) K. Schum.), they were also not certain that the characters given to separate the two genera would hold up to further investigation.

In my opinion, despite the diversity in fruit type the species are all very similar in flower and vegetative characters. Other clear characters are more useful for delimiting species rather than genera or sections. On the other hand the fruit difference is so clear and occurs so constantly that it should not be ignored. For that reason I prefer to treat them as sections under the genus Ochrosia Juss. until further evidence from a more comprehensive study is obtained and the relationship between these 2 sections becomes clearer.

Here, I apply the names Ochrosia section Echinocaryon F. Muell. (the oldest name at section level for the fibrous-fruited species) and Ochrosia section Ochrosia for the fibrous-fruited species and cavity-fruited species of Ochrosia, respectively, and reduce Neisosperma into Ochrosia section Echinocaryon F. Muell.

The name Ochrosia section Lactaria used by Mueller (1871), Valeton (1895) (as subgenus) and Pichon (1947) for the cavity-fruited species cannot be applied since it does not comply with the provisions of Article 22 of the International Code of Botanical Nomenclature (Greuter et al., 2000), and Ochrosia section Ochrosia is the valid name.

\section{Malesian Ochrosia}

Ochrosia species are widespread in mostly coastal areas and lowland forests in Africa, South Asia, Indochina, Malesia, northern Australia, and the Pacific Islands. In Malesia, there are 13 species, including $O$. syncarpa Markgr. (the only species with syncarpous fruit), and $O$. ackeringae (Teijsm. \& Binn.) Miq. (the only species with hemisyncarpous fruit).

Ochrosia oppositifolia (Lam.) K. Schum. is the species with the widest distribution, found from the Seychelles in the west, through the Maldive Islands, Andaman, 
Sri Lanka, Vietnam, Thailand, Malesia, and the Pacific Islands. Some others, such as O. acuminata Trimen ex Valeton, O. apoensis Elmer, O. minima (Markgr.) Fosberg \& Boiteau, $O$. syncarpa Markgr. and $O$. tenimberensis Markgr., are known only from restricted areas.

\section{MATERIAL AND METHODS}

Herbarium material was studied from the following herbaria: A, BM, BO, CANB, GH, K, L, M, NSW, P, UC, WAG, and WRSL.

The dimensions given in the descriptions are for dried material except for the gynoecium and androecium characters which are for flowers rehydrated with water.

\section{OCHROSIA Juss.}

Ochrosia Juss. (1789) 144; A.DC. (1844) 356; Seem. (1865) 158; F. Muell. (1871) 130; Valeton (1895) 225; Markgr. (1927) 189; Pichon (1947) 205, 206; Leeuwenb. (1987) 47. - Ochrosia Juss. sect. Ochrosia. - Ochrosia Juss. sect. Lactaria F. Muell. (1871) 130; Pichon (1947) 206. - Ochrosia Juss. subgen. Lactaria (F. Muell.) Valeton (1895) 225. - Ochrosia sensu Fosberg \& Sachet (1972) 47; Fosberg \& Sachet (1974) 254; Fosberg, Boiteau \& Sachet (1977) 23; Markgr. (1979) 233; Boiteau \& L. Allorge (1981) 47. - Type: Ochrosia maculata Jacq. (= Ochrosia borbonica J.F. Gmel., nom. nud.).

Neisosperma Raf. (1838) 162; Fosberg \& Sachet (1972) 48 (as 'Neiosperma'); (1974) 254, 255 (as 'Neiosperma'); Fosberg, Boiteau \& Sachet (1977) 28; Markgr. (1979) 241; Boiteau \& L. Allorge (1981) 64; P.I. Forst. (1993) 14; M.E. Endress \& Bruyns (2000) 34. - Lectotype: Neisosperma muricata Raf. (= N. oppositifolia $($ Lam.) Fosberg \& Sachet = Ochrosia oppositifolia $($ Lam.) K. Schum.), designated by Markgraf, 1979.

Lactaria Rumph. ex Raf. (1838) 162, nom. illeg.; Koidz. (1923) 48 (excl. L. borbonica J.F. Gmel. = Ochrosia borbonica J.F. Gmel.). - Type: Lactaria salubris Rumph. ex Raf. (= Ochrosia oppositifolia (Lam.) K. Schum.).

Pseudochrosia Blume (1850) 158; K. Schum. (1895) 156. - Type: Pseudochrosia glomerata Blume (= Ochrosia glomerata (Blume) F. Muell.).

Bleekeria Hassk. emend. Koidz. (1923) 51. - Type: Bleekeria kalokarpa Hassk. (= Ochrosia elliptica Labill.).

Excavatia Markgr. (1927) 194; (1936) 127. - Lectotype: Excavatia littoralis (Merr.) Markgr. (= Ochrosia ackeringae (Teijsm. \& Binn.) Miq.), designated by Leeuwenberg, 1987.

Calpicarpum G. Don emend. Boiteau in Boiteau et al. (1974) 495, nom. illeg.; Boiteau, L. Allorge \& Sevenet (1975) 152. - Lectotype: Calpicarpum roxburghii G. Don (= Kopsia fruticosa (Roxb.) A.DC.), designated by De Candolle, 1844.

Ochrosia sect. Echinocaryon F. Muell. (1871) 130; Pichon (1947) 209. - Ochrosia subg. Echinocaryon Valeton (1895) 225. - Type species: Ochrosia kilneri F. Muell.

Ochrosia sect. Phragmochrosia Pichon (1947) 211. - Type: Ochrosia apoensis Elmer.

Trees or shrubs with white latex. Branchlets terete, subangular or angular, glabrous, not lenticellate. Leaves 2-4(-6)-whorled, papyraceous to coriaceous when dried, glabrous, petiolate; blades obovate, elliptic, or narrowly so, midrib prominent on abaxial side, impressed on adaxial side, glabrous; secondary veins usually rather straight, rarely strongly arcuate ascending, not reaching the margin, usually joining and forming a submarginal vein, rarely not joining. Inflorescences terminal and axillary cymes, in whorls or solitary, loose or congested, few- to many-flowered, glabrous, often with bracts, pedunculate; peduncle varying considerably in length, sometimes winged (in Malesia in 2 species only). Flowers sometimes fragrant, small, pedicellate; pedicel sepal-like, 
bracteolate. Sepals ovate, or suborbicular to orbicular, margin entire or ciliolate, apex rounded or acute, without colleters inside, connate at base. Corolla salverform, white, greenish white, creamy white, or yellowish white, villose inside, glabrous outside; lobes elliptic, obovate, narrowly elliptic, or narrowly ovate, overlapping to the right, glabrous. Stamens included, free from each other; cells parallel, dehiscent throughout by longitudinal slits; filaments filiform, short. Ovary of 2 carpels (which are usually distinctly separated), gradually or abruptly narrowed at apex towards base of style, glabrous; disc entire, small; style filiform, split or not at base. Fruits usually apocarpous, rarely hemisyncarpous or syncarpous, drupaceous, smooth or remotely verrucose to verrucose, or with fine irregular ridges when dried, green and turning yellow, orange, red, or purple when ripe; mericarps ellipsoid, obovoid, ovoid, or subglobose, rarely (not in Malesian species) discoid or boat-shape, round in transverse section, or dorsoventrally compressed, sometimes with lateral and/or apical ridges, or with lateral and/or apical wing (not in Malesian species); endocarps split into diverging fibres, or consisting of thick and massive tissue, surrounding 2 lateral cavities. Seeds 1-3 on each placenta, flat, suborbicular or elliptic, with a wing-like structure along the margin, glabrous.

Distribution - About 40 species, of which 13 in Malesia. The majority of species are in the Pacific Islands. Spread from the Mascarene and Seychelles in the west throughout South Asia, Indochina, Malesia, northern Australia, and Pacific Islands as far east as the Marquesas and Hawaiian Islands.

\section{KEY TO THE SECTIONS}

1a. Fruits apocarpous; mericarps verrucose to smooth, or with fine irregular ridges when dried, more or less round in transverse section, not compressed; endocarps split into diverging fibres, lateral cavities absent. . Ochrosia sect. Echinocaryon

b. Fruits apocarpous, rarely hemisyncarpous, or syncarpous; mericarps often smooth when dried, often dorsoventrally compressed or biconvex in transverse section; endocarps not split into diverging fibres, hard, thick, massive, surrounding 2 lateral cavities ...................... Ochrosia sect. Ochrosia

\section{KEY TO THE MALESIAN SPECIES}

Based on flowering and fruiting material

1a. Secondary veins arcuate ascending, not more than 20 pairs, not joining, not forming

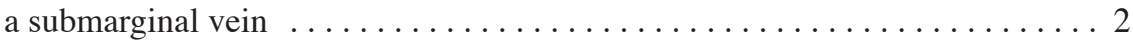

b. Secondary veins straight to rather arcuate ascending, 10 to more than 40 pairs, joining, forming a submarginal vein $\ldots \ldots \ldots \ldots \ldots \ldots \ldots \ldots \ldots \ldots \ldots$

2a. Leaves 2-4-whorled, blades papyraceous. Inflorescence 4-9 cm long. Sepals ovate; stamens inserted at $0.7-0.8$ of the length of the corolla tube 4. O. ficifolia

b. Leaves 4-6(-7)-whorled, blades coriaceous. Inflorescence 9.5-18.5 cm long. Sepals suborbicular to orbicular. Stamens inserted at 0.2 of the length of the corolla

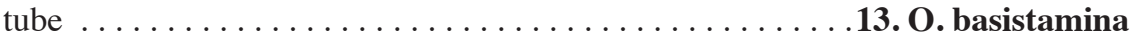

3a. Leaf blades width $1-1.7 \mathrm{~cm}$, ratio $4.2-7.1$. Fruits apocarpous or hemisyncarpous . Leaf blades width $2.1-18 \mathrm{~cm}$, ratio $1-5.8$. Fruit syncarpous . . 11. O. syncarpa 
4a. Leaf blades papyraceous when dried. Peduncle winged $\ldots \ldots \ldots \ldots \ldots \ldots$

b. Leaf blades coriaceous or papyraceous when dried. Peduncle not winged (unknown

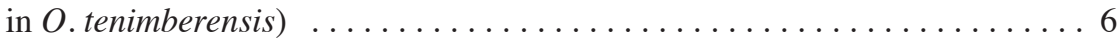

5a. Leaf apex acute or acuminate; peduncle 3.5-6 cm long; corolla tube 3.9-4 mm

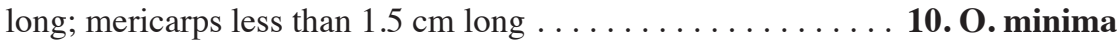

b. Leaf apex cuspidate; peduncle $0.6-3.3 \mathrm{~cm}$ long; corolla tube $13 \mathrm{~mm}$ long; mericarps up to $4.6 \mathrm{~cm}$ long $\ldots \ldots \ldots \ldots \ldots \ldots \ldots \ldots \ldots \ldots \ldots \ldots \ldots$ 9. O. coccinea

6a. Calyx not ciliolate at margin (unknown in O.tenimberensis and $O$. sciadophylla).

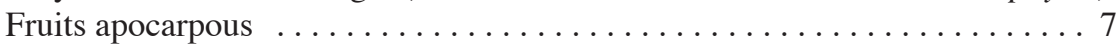

b. Calyx ciliolate at margin. Fruits hemisyncarpous . . . . 8. O. ackeringae

7a. Mericarps verrucose to remotely so, or with fine irregular ridges when dried, endocarps loosely fibrous, lateral cavities absent $\ldots \ldots \ldots \ldots \ldots \ldots$

b. Mericarps smooth when dried, endocarps massive, hard, surrounding 2 lateral cavities

12. O. tenimberensis

8a. Secondary veins infrequently anastomosing around the submarginal vein, submarginal veins not angled, close to the margin. Fibres of the endocarps rather slender and thin (except for O. oppositifolia, which are slender and thin or hard and thick). Seeds 1 or 2 at either placenta . . . . . . . . . . . 9

b. Secondary veins frequently anastomosing around the submarginal vein, submarginal vein angled, $0.3-1 \mathrm{~cm}$ inside from the margin. Fibres of the endocarps hard and thick. Seeds 3 at either placenta ............... citrodora

9a. Secondary veins less than 17 pairs. Mericarps $2.8-4.7 \mathrm{~cm}$ long . . . . . . 10

b. Secondary veins 20 to more than 40 pairs. Mericarps $4.1-8.5 \mathrm{~cm}$ long . . . . 11

10a. Leaf apex acute to abruptly acuminate, or sometimes rounded. Peduncle 1-3 $\mathrm{cm}$ long. Corolla tube $6 \mathrm{~mm}$ long; sepals ovate, apex rounded, thick. Mericarps apiculate at apex $\ldots \ldots \ldots \ldots \ldots \ldots \ldots \ldots \ldots \ldots \ldots \ldots \ldots \ldots \ldots$. Ocuminata

b. Leaf apex gradually acuminate to cuspidate. Peduncle $0.2-1 \mathrm{~cm}$ long; corolla tube 7.5-8 mm long; sepals orbicular, apex obtuse, thin. Mericarps acuminate at

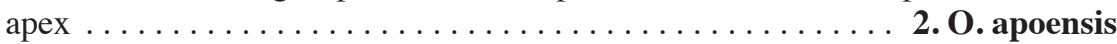

11a. Secondary veins straight to rather arcuate ascending near the margin, 21 to more than 40 pairs. Flowers more than 30 in each inflorescence. Seeds 1 or 2 at either

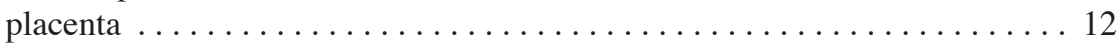

b. Secondary veins arcuate ascending, 20-22 pairs. Flowers 10-12 in each inflorescence. Seeds 1 at either placenta $\ldots \ldots \ldots \ldots \ldots \ldots$ 7. O. sciadophylla

12a. Leaf blades ratio $2.1-5.8$, secondary veins $0.1-0.7 \mathrm{~cm}$ spaced. Corolla tube 3 $\mathrm{mm}$ long. Stamens inserted at $1 \mathrm{~mm}$ below the mouth. Seeds always 2 at either

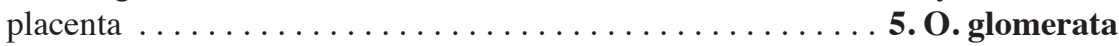

b. Leaf blades ratio $1-3$, secondary veins $0.2-1.5 \mathrm{~cm}$ spaced. Corolla tube $5.3-7$ $\mathrm{mm}$ long. Stamens inserted at $2-2.5 \mathrm{~mm}$ below the mouth. Seeds often 1 at either placenta (or rarely with a small second one)

6. O. oppositifolia

\section{OCHROSIA section ECHINOCARYON}

Ochrosia sect. Echinocaryon F. Muell. (1871) 130; Pichon (1947) 209. - Ochrosia subg. Echinocaryon Valeton (1895) 225. - Type: Ochrosia kilneri F. Muell.

Neisosperma Raf. (1838) 162; Fosberg \& Sachet (1972) 48 (as 'Neiosperma'); (1974) 254, 255 (as 'Neiosperma'); Fosberg, Boiteau \& Sachet (1977) 28; Markgr. (1979) 241; Boiteau \& L. Allorge 
(1981) 64; P.I. Forst. (1993) 14; M.E. Endress \& Bruyns (2000) 34. - Lectotype: Neisosperma muricata Raf. (= N. oppositifolia (Lam.) Fosberg \& Sachet = Ochrosia oppositifolia (Lam.) K. Schum.), designated by Markgraf, 1979.

Lactaria Rumph. ex Raf. (1838) 162, nom. illeg.; Koidz. (1923) 48 (excl. L. borbonica J.F. Gmel. = Ochrosia borbonica J.F. Gmel.). - Type: Lactaria salubris Rumph. ex Raf. (= Ochrosia oppositifolia (Lam.) K. Schum.).

Pseudochrosia Blume (1850) 158; K. Schum. (1895) 156. - Type: Pseudochrosia glomerata Blume (= Ochrosia glomerata (Blume) F. Muell.).

Ochrosia sensu Markgr. (1927) 189.

Ochrosia sect. Phragmochrosia Pichon (1947) 211. - Type: Ochrosia apoensis Elmer.

Calpicarpum G. Don emend. Boiteau in Boiteau et al. (1974) 495, nom. illeg.; Boiteau, L. Allorge \& Sevenet (1975) 152. - Lectotype: Calpicarpum oppositifolium (Lam.) Boiteau, designated by Boiteau in Boiteau et al., 1974. See note below.

Fruits apocarpous. Mericarps verrucose to remotely so, or with fine irregular ridges when dried, more or less round in transverse section, not compressed. Endocarps split into diverging fibres, lateral cavities absent.

Note - Alphonse de Candolle (1844) reduced Calpicarpum G. Don to Kopsia Blume, and excluded C. lamarckii G. Don (= Cerbera oppositifolia Lam. = Ochrosia oppositifolia (Lam.) K. Schum.). It was supported by Fosberg \& Sachet (1977), who showed clearly that the description in the protologue of Calpicarpum G. Don almost entirely applies to Kopsia. However, Boiteau et al.(1974, 1975) ignored it and applied this name (Calpicarpum) to the component they were segregating from Ochrosia, exemplified by the plant excluded by De Candolle. Boiteau in Boiteau et al. (1974) designated Calpicarpum oppositifolium (Lam.) Boiteau as the type species for his Calpicarpum.

\section{KEY TO THE MALESIAN SPECIES OF OCHROSIA SECTION ECHINOCARYON}

1a. Style split at the base. Mericarps acute to acuminate at apex, fibres hard and thick, forming hard thick pointed ends. Seeds 3 at either placenta . . . . . . . 2

b. Style not split at the base (unknown in $O$. sciadophylla). Mericarps acuminate to apiculate at apex, fibres rather soft and slender, not forming hard thick pointed ends (except $O$. oppositifolia, which are slender and thin or hard and thick). Seeds 1 or

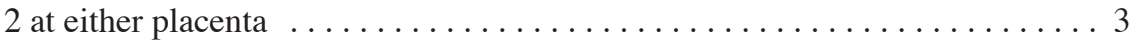

2a. Secondary veins strongly arcuate ascending, less than 15 pairs, not joining, submarginal vein absent. Style 7-9 mm long. Mericarps narrowly ellipsoid or narrowly

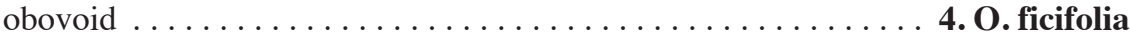

b. Secondary veins straight, 15-32 pairs, joining, submarginal vein present. Style $3-5.1 \mathrm{~mm}$ long. Mericarps ellipsoid .............. citrodora

3a. Secondary veins 10-16 pairs. Ovary gradually narrowed at apex towards base of style. Mericarps with lateral ridges $\ldots \ldots \ldots \ldots \ldots \ldots \ldots \ldots \ldots$

b. Secondary veins 20 to more than 40 pairs. Ovary rounded at apex and abruptly narrowed at base of style (unknown in O. sciadophylla). Mericarps without lateral

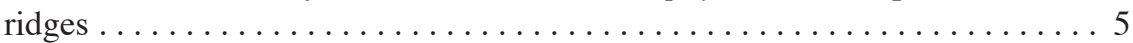

4a. Leaf blades acute to abruptly acuminate at apex, sometimes rounded. Peduncle 1-3 $\mathrm{cm}$ long. Sepals ovate, apex rounded, thick. Corolla tube $6 \mathrm{~mm}$ long, tube-lobes ratio 0.9 . Mericarps apiculate at apex, acumen $0.4-1 \mathrm{~cm}$ long . 1. O. acuminata 
b. Leaf blades gradually acuminate to cuspidate at apex. Peduncle $0.2-1 \mathrm{~cm}$ long . Corolla tube 7.5-8 mm long, tube-lobes ratio 1.3-1.5. Sepals suborbicular to orbicular, apex obtuse, thin. Mericarps acuminate at apex, acumen less than $0.5 \mathrm{~cm}$

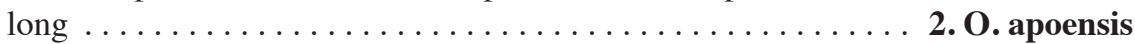

5a. Secondary veins straight to rather arcuate ascending near the margin, 21 to more than 40 pairs. Flowers more than 30 on each inflorescence . . . . . . . . 6

b. Secondary veins arcuate ascending, 20-22 pairs. Flowers $10-12$ on each inflorescence $\ldots \ldots \ldots \ldots \ldots \ldots \ldots \ldots \ldots \ldots \ldots \ldots \ldots \ldots \ldots \ldots \ldots \ldots$. $\ldots_{\ldots}$ sciadophylla

6a. Corolla tube $3 \mathrm{~mm}$ long; stamens inserted at $1 \mathrm{~mm}$ below the mouth. Mericarps verrucose when dried; seeds always 2 at either placenta . . . 5. O.glomerata

b. Corolla tube 5.3-7 mm long; stamens inserted at 2-2.5 mm below the mouth Mericarps remotely verrucose when dried; seeds often 1 at either placenta (or rarely with a small second one) $\ldots \ldots \ldots \ldots \ldots \ldots \ldots \ldots \ldots$. O. oppositifolia

\section{Ochrosia acuminata Trimen ex Valeton}

Ochrosia acuminata Trimen ex Valeton (1895) 231, pl. xxii; Pichon (1947) 209. - Neisosperma acuminata (Trimen ex Valeton) Fosberg \& Sachet (1977) 28. - Neisosperma acuminata (Trimen ex Valeton) Fosberg \& Sachet var. acuminata; Markgr. (1979) 244. - Lactaria acuminata (Trimen ex Valeton) Koidz. (1923) 51. - Type: Teijsmann s.n. (holo n.v.; iso L), Indonesia, Sulawesi, North Sulawesi, Menado.

Tree, (8-)15-30 m high. Branchlets subangular to angular, 3-5 mm diam., smooth, not lenticellate, glabrous, sometimes with conspicuous leaf scars. Leaves 3- or 4-whorled, coriaceous when dried, petiolate; petiole 1-2 cm long, glabrous; blades usually obovate, rarely narrowly so, $5.7-16(-23)$ by $2.7-6.6 \mathrm{~cm}$, ratio $1.7-2.9(-3.3)$, base cuneate, margin entire to undulate, apex acute or abruptly acuminate, sometimes rounded; secondary veins conspicuous, usually prominent, $10-14$ pairs, $0.5-2 \mathrm{~cm}$ spaced, at an angle of $45-60^{\circ}$ to the midrib, straight to strongly arcuate ascending, not reaching the margin, joining, forming a submarginal vein which is $1-2 \mathrm{~mm}$ inside from the margin, often branched before reaching the submarginal vein; submarginal vein sometimes inconspicuous; tertiary veins not prominent, rarely conspicuous. Inflorescence 2.2-6 $\mathrm{cm}$ long, axillary cymes, in whorls of 2 or 3 , loose, glabrous, with bracts, pedunculate; peduncle 1-3 cm long, glabrous, thick. Flowers 10-15(-25) on each inflorescence; pedicels $2.5 \mathrm{~mm}$ long, glabrous, bracteolate; bracteoles sepal-like, ovate, 1 by $1 \mathrm{~mm}$, margin entire, apex acute, thin, glabrous on both sides, 2 or 3 on each pedicel. Sepals ovate, $1.7-2$ by $1.4-2 \mathrm{~mm}$, ratio 1-1.2, margin entire, apex rounded, glabrous on both sides, thick, spreading outwards, connate at base for $0.5 \mathrm{~mm}$. Corolla greenish cream or pale yellow, in mature buds narrowly ellipsoid, $9-10$ by $2 \mathrm{~mm}$, ratio $4.5-5$, of which the lobes form a rounded apex, glabrous outside, villose inside, rather dense, forming a belt of $1.5 \mathrm{~mm}$ wide just below the insertion of stamens; tube cylindrical, $6 \mathrm{~mm}$ long, straight, widening at the stamens insertion, and there $2 \mathrm{~mm}$ wide, tube-calyx ratio 3-3.5, tube-lobes ratio 0.9; lobes elliptic, 6.5 by $3 \mathrm{~mm}$, ratio 2.2, margin entire, apex obtuse, glabrous, auriculate on the left side at base. Stamens inserted at $3 \mathrm{~mm}$ below the mouth, at 0.5 of the length of the corolla tube; filaments filiform, $0.8 \mathrm{~mm}$ long; anthers ovate, 1.3 by $0.6 \mathrm{~mm}$, sagittate at base, apex acute, yellowish when dried. Pistil glabrous, $4.7 \mathrm{~mm}$ long; ovary ovoid, widening at base, gradually narrowed at apex towards base of style, 0.9 by $0.8 \mathrm{~mm}$, of 2 distinctly separated carpels; style filiform, 
$3 \mathrm{~mm}$ long, style-ovary ratio 3.3, not split at base; pistil head conical, 0.8 by $0.3 \mathrm{~mm}$. Fruits composed of 2 separate mericarps; mericarps blackish brown when dried, ellipsoid, $2.9-4.7$ by $1.5-2.5$ by $1.5-2.5 \mathrm{~cm}$, base rounded, apex gradually apiculate, acumen $0.4-1 \mathrm{~cm}$ long, straight or rather curled, with distinct lateral ridges, remotely verrucose or with fine irregular ridges when dried, glabrous, rather glossy, indehiscent; endocarps split into fibres penetrating the mesocarps; fibres rather thin and slender, not forming hard thick pointed ends. Seeds 1 at either placenta, flat, ellipsoid, 1-3 by $0.8-1.7 \mathrm{~cm}$, apex acute, glabrous.

Distribution - Malesia: Indonesia (Sulawesi).

Habitat \& Ecology - Found in disturbed primary forests, open secondary forests, or open areas such as roadsides. On clayey or sandy soil. Altitude 0-1500 m.

Notes -1 . Holotype specimen is not found. It was probably in BO.

2. This species is characterized by the long acumen on the fruits.

3. Markgraf (1979) included two varieties in this species, namely Neisosperma acuminata var. acuminata and N. acuminata var. apoense. Var. apoense is here treated as a separate species.

\section{Ochrosia apoensis Elmer}

Ochrosia apoensis Elmer (1912) 1461; Merr. (1923) 329; Pichon(1947) 211. - Neisosperma apoensis (Elmer) Fosberg \& Sachet (1977) 28. - Neisosperma acuminata (Trimen ex Valeton) Fosberg \& Sachet var. apoense (Elmer) Markgr. (1979) 244. - Type: Elmer 10478 (holo PNH†; lecto L, designated here; iso A), Philippines, Mindanao, Davao, Todaya-Mt Apo.

Tree, 10-21 $\mathrm{m}$ high. Branchlets terete, subangular to angular, 3-5 mm diam., smooth, not lenticellate, glabrous, sometimes with conspicuous leaf scars. Leaves 3- or 4whorled, coriaceous when dried, petiolate; petiole 1-2.5 cm long, glabrous; blades (narrowly) obovate, $7-14$ by $2.2-4.2 \mathrm{~cm}$, ratio $2.7-4.7$, base cuneate, margin entire to undulate, apex gradually acuminate to cuspidate; secondary veins conspicuous on both sides, rather prominent on abaxial side, $13-16$ pairs, $0.3-1 \mathrm{~cm}$ spaced, at an angle of $70-85^{\circ}$ to the midrib, straight to rather arcuate ascending near the margin, not reaching the margin, joining, forming a submarginal vein which is $1-2 \mathrm{~mm}$ inside from the margin; tertiary veins not prominent, conspicuous on abaxial side only, reticulate. Inflorescence $1.5-3.5 \mathrm{~cm}$ long, axillary cymes, in whorls of 2 or 3, loose, glabrous, bracteolate, shortly pedunculate; peduncle $0.2-1 \mathrm{~cm}$ long, glabrous. Flowers $7-10$ in each inflorescence; pedicels $2 \mathrm{~mm}$ long, glabrous, bracteolate; bracteoles sepal-like, ovate, $1-1.1$ by $1-1.7 \mathrm{~mm}$, margin entire, apex acute, thin, glabrous on both sides, 3 or 4 on each pedicel. Sepals suborbicular to orbicular, $1.8-2$ by $2-2.7 \mathrm{~mm}$, ratio $0.7-1$, margin entire, apex obtuse, glabrous on both sides, thin, rather transparent, not spreading outwards, connate at base for $0.6 \mathrm{~mm}$. Corolla white, in mature buds narrowly ellipsoid, 14 by $2 \mathrm{~mm}$, ratio 7, of which the lobes form a rounded apex, glabrous outside, villose inside, rather dense, forming a belt of $1.5 \mathrm{~mm}$ wide just below the insertion of stamens; tube cylindrical, 7.5-8 $\mathrm{mm}$ long, straight, widening at the stamens insertion, and there $2 \mathrm{~mm}$ wide, tubes-calyx ratio 4-4.2, tubes-lobes ratio 1.3-1.5; lobes elliptic, $5-6$ by $3 \mathrm{~mm}$, ratio 1.7-2, margin entire, apex obtuse, glabrous, thin, auriculate on the left side at base. Stamens inserted at $3.5 \mathrm{~mm}$ below the mouth, 0.5 of the length of the corolla tube; filaments filiform, $0.5 \mathrm{~mm}$ long; anthers ovate, 1.5 by $0.5 \mathrm{~mm}$, sagittate at base, apex acute, yellowish when dried. Pistil glabrous, $5 \mathrm{~mm}$ long; ovary ovoid, 
widening at base, gradually narrowed at apex towards base of style, 1 by $0.8 \mathrm{~mm}$, of 2 distinctly separated carpels; style filiform, $3.4 \mathrm{~mm}$ long, style-ovary ratio 3.4, not split at base; pistil head conical, $0.4-0.5$ by $0.3 \mathrm{~mm}$. Fruits composed of 2 separate mericarps; mericarps blackish when dried, ellipsoid, $2.8-3.6$ by $1.4-2$ by $1.4-2 \mathrm{~cm}$, base rounded, apex acuminate, acumen short, less than $0.5 \mathrm{~cm}$ long, straight or rather curled, with distinct lateral ridges, remotely verrucose or with fine irregular ridges when dried, rather glossy, glabrous, indehiscent; endocarps split into fibres penetrating the mesocarps; fibres rather thin and slender, not forming hard thick pointed ends. Seeds 1 at either placenta, flat, ellipsoid, $1-2.2$ by $0.8-1 \mathrm{~cm}$, apex acute, glabrous.

Distribution - Malesia: Philippines (Luzon, Mindanao)

Habitat \& Ecology - Forest slopes. Altitude 810-970 m.

Notes - 1. Markgraf (1979) included this species as a variety of Neisosperma acuminata (Trimen ex Valeton) Fosberg \& Sachet (=O. acuminata Trimen ex Valeton). However, I agree with Elmer (1912) who mentioned that this species resembles O. acuminata but is sufficiently distinct to deserve specific status. The key to the species gives the differences between the two. The clearest one is a sepal character (sepals ovate and thick vs. orbicular and thin).

2. In general, the leaf blades are narrower than those of $O$. acuminata.

\section{Ochrosia citrodora Lauterb. \& K. Schum.}

Ochrosia citrodora Lauterb. \& K. Schum. in K. Schum. \& Lauterb. (1901) 504; Markgr. (1927) 190; Pichon (1947) 211 (as 'O. citriodora'). - Neisosperma citrodora (Lauterb. \& K. Schum.) Fosberg \& Sachet (1977) 29; Markgr. (1979) 245. - Type: Lauterbach 1073 (holo B†; lecto WRSL, designated by Markgraf, 1979), Papua New Guinea, Madang, Gogol.

Paralstonia clusiacea auct. non Baill.: Markgr. (1926) 283. - The specimen mentioned (Gjellerup 116 ) is clearly $O$. citrodora.

Shrub or tree, 2-25 m high. Branchlets angular to subangular, 3-6 mm diam., not lenticellate, smooth, glabrous, with conspicuous leaf scars. Leaves 2- or 3-whorled, coriaceous or papyraceous when dried, petiolate; petiole $0.7-3.5 \mathrm{~cm}$ long, glabrous; blades (narrowly) obovate or (narrowly) elliptic, $9.5-29.5$ by $3.1-8.3 \mathrm{~cm}$, ratio $1.9-3.8$, base cuneate, margin entire, rarely undulate, apex acuminate or cuspidate; secondary veins usually inconspicuous, not prominent, $15-32$ pairs, $0.2-0.7 \mathrm{~cm}$ spaced, at an angle of $80-90^{\circ}$ to the midrib, straight to rather arcuate ascending near the margin, not reaching the margin, joining, forming a submarginal vein, much anastomosing around the submarginal vein; submarginal vein usually angled, $0.3-1 \mathrm{~cm}$ inside from the margin; tertiary veins not prominent, conspicuous, distinctly reticulate especially around submarginal vein. Inflorescence $4-10.5 \mathrm{~cm}$ long, terminal and axillary cymes, in whorls of 2 or 3 , loose, glabrous, sometimes with bracts, pedunculate; peduncle $2.5-8 \mathrm{~cm}$ long, glabrous, often slender. Flowers 5-17(-30) in each inflorescence, fairly long pedicellate; pedicels 3.5-5 mm long, glabrous, bracteolate; bracteoles sepal-like, ovate, $1-1.3$ by $1-1.1 \mathrm{~mm}$, margin entire, apex rounded, rough outside, smooth inside, glabrous, $2-7$ on each pedicel. Sepals ovate, $2.1-3.2$ by $1.8-2.2 \mathrm{~mm}$, ratio $1.1-1.8$, margin entire, apex rounded, thick except along the margin, glabrous on both sides, rough outside, smooth inside, connate at base for $1 \mathrm{~mm}$. Corolla white, creamy white, yellowish white, or greenish white, in mature buds narrowly ellipsoid, $13-18$ by $3 \mathrm{~mm}$, ratio 4.3-6, of which the lobes form a rounded apex, glabrous outside, villose inside, 
rather dense, forming a belt of 1.5-2 mm wide just below the insertion of stamens; tube cylindrical, 11-12 mm long, straight, widening at the stamens insertion, and there $2-2.3 \mathrm{~mm}$ wide, tube-calyx ratio 3.4-5.2, tube-lobes ratio 0.9-1.2; lobes narrowly elliptic, $9-14$ by $3 \mathrm{~mm}$, ratio 3-4.7, margin entire, apex rounded, glabrous, auriculate on the left side at base. Stamens inserted at 3.5-4 mm from the mouth, 0.6-0.7 of the length of the corolla tube; filaments filiform, $0.5 \mathrm{~mm}$ long; anthers ovate to narrowly so, 2 by $0.5-0.8 \mathrm{~mm}$, sagittate at base, apex acute, yellowish when dried. Pistil glabrous, 4.3-7.1 mm long; ovary subglobose, 1 by $1 \mathrm{~mm}$, of 2 distinctly separated carpels, widening at base, apex rounded and abruptly narrowed at base of style; style filiform, 3-5.1 mm long, style-ovary ratio 2.4-5.1, split at base; pistil head ovoid, $0.8-0.9 \mathrm{~mm}$ long. Fruits composed of 2 separate mericarps; mericarps brown or reddish brown when dried, ellipsoid, $5.4-9.6$ by $1.8-3.8$ by $1.3-3.2 \mathrm{~cm}$, base cuneate or rounded, apex acute, remotely verrucose when dried, glabrous, indehiscent, green and turning yellow, orange, or purplish red when ripe; endocarps split into fibres penetrating the mesocarps; fibres hard and thick, usually forming hard thick pointed ends. Seeds 3 at either placenta, flat, suborbicular to ellipsoid, unequal in size, the first two $0.8-3.1$ by $0.7-2.5$, the second one $0.6-1.3$ by $0.4-1.2 \mathrm{~cm}$, apex acuminate, glabrous, whitish brown.

Distribution - Malesia: Indonesia (West Papua), Papua New Guinea.

Habitat \& Ecology - In primary and secondary lowland rain forests, gully forests, riversides, or swampy areas. On clay, stony soil, or copper-rich soil. Altitude $0-850 \mathrm{~m}$.

Note - Characterized by its secondary veins, which are frequently anastomosing around the submarginal vein, and its angled submarginal vein, which is situated 3-10 $\mathrm{mm}$ inside from the margin.

\section{Ochrosia ficifolia (S. Moore) Markgr.}

Ochrosia ficifolia (S. Moore) Markgr. (1927) 190; Pichon (1947) 211. - Alstonia ficifolia S. Moore (1923) 32. - Neisosperma ficifolia (S. Moore) Fosberg \& Sachet in Fosberg, Boiteau \& Sachet (1977) 29; Markgr. (1979) 244. - Type: Forbes 74 (holo BM; iso L), Papua New Guinea, Central, Sogeri.

Ochrosia rudis Markgr. (1927) 189; Pichon (1947) 211. - Neisosperma rudis (Markgr.) Fosberg \& Sachet in Fosberg, Boiteau \& Sachet (1977) 31. - Type: Beccari 715 (holo FI; photo in L), Papua New Guinea, Andai.

Shrub or tree, 3-30 $\mathrm{m}$ high. Branchlets angular to subangular, thick, 4.5-7 mm diam., sulcate when dried, not lenticellate, smooth, glabrous. Leaves 2-4-whorled, papyraceous when dried, sometimes thickly so; petiole 1-4 cm long, glabrous; blades (narrowly) obovate, $10.5-26$ by $4.6-10 \mathrm{~cm}$, ratio $1.9-3.9$, base cuneate to long cuneate, margin entire, apex acuminate to cuspidate, rarely acute; secondary veins conspicuous, slightly prominent on abaxial side, $7-12$ pairs, $0.8-3.1 \mathrm{~cm}$ spaced, at an angle of c. $90^{\circ}$ to the midrib, strongly arcuate ascending, not reaching the margin, not joining, not forming a submarginal vein; tertiary veins indistinct. Inflorescence 4-9 cm long, axillary cymes, in whorls of 2, not much branched, congested, glabrous, sometimes with bracts, pedunculate; peduncle 3-7 cm long, thick, glabrous. Flowers 20-25 on each inflorescence, pedicellate; pedicels 1-4 mm long, glabrous, bracteolate; bracteoles sepal-like, ovate, $1-1.3$ by $0.7-1.3 \mathrm{~mm}$, margin entire, apex rounded, rough outside, smooth inside, glabrous, 2 or 3 on each pedicel. Sepals ovate, $2.2-3$ by $1.4-1.9 \mathrm{~mm}$, 
ratio 1.4-1.6, margin entire, apex rounded, thick except along the margin, glabrous on both sides, rough outside, smooth inside, connate at base for $1 \mathrm{~mm}$. Corolla white, cream, yellow, in mature buds narrowly ellipsoid, $13-16$ by $3 \mathrm{~mm}$, ratio $4.3-5.3$, of which the lobes form a rounded apex, glabrous outside, villose inside, rather dense, forming a belt of 2-2.5 mm wide just below the insertion of stamens; tube cylindrical, 11-14.3 mm long straight, widening at the stamens insertion, and there 2-2.5 $\mathrm{mm}$ wide, tube-calyx ratio $4.8-5.1$, tube-lobes ratio $0.8-0.9$; lobes narrowly elliptic, $13-16$ by $4-5 \mathrm{~mm}$, ratio $3.2-4$, margin entire, apex rounded, glabrous, auriculate on the left side at base. Stamens inserted at 3.5-4 mm below the mouth, 0.7-0.8 of the length of the corolla tube; filaments filiform, $0.3 \mathrm{~mm}$ long; anthers ovate to narrowly so, $1.5-1.6$ by $0.5 \mathrm{~mm}$, sagittate at base, apex acute, yellowish when dried. Pistil glabrous, $8.8-9 \mathrm{~mm}$ long; ovary subglobose, $0.9-1$ by $0.9 \mathrm{~mm}$, of 2 distinctly separated carpels, widening at base, apex rounded and abruptly narrowed at base of style; style filiform, 7-9 mm long, style-ovary ratio 7-9, split at base; pistil head ovoid, 0.8-0.9 mm long. Fruits composed of 2 separate mericarps; mericarps brown or blackish brown when dried, narrowly ellipsoid to narrowly obovoid, $7.2-11$ by $2.6-3$ by $2.5-2.7 \mathrm{~cm}$, base rounded, apex acuminate, acumen short, remotely verrucose or with fine irregular ridges when dried, glabrous, indehiscent, green and turning purple, or bluish purple when ripe; endocarps split into fibres penetrating the mesocarps; fibres hard and thick, usually forming hard thick pointed ends. Seeds 3 at either placenta, flat, suborbicular to ellipsoid, apex acuminate, glabrous, brown, unequal in size, the first two 1.8-2.4 by $1.5-1.6 \mathrm{~cm}$, the third $0.5-1.6$ by $0.4-1.1 \mathrm{~cm}$.

Distribution - Malesia: Indonesia (West Papua), Papua New Guinea.

Habitat \& Ecology - Mostly in primary lowland forests. Also found in monsoon forests, swampy forests, floodplain forests or dry gullies on margin of seasonally arid grassland. On clayey, sandy, stony, or gravely clayey soil. Altitude $2-800(-2100) \mathrm{m}$.

Notes -1 . The species usually occurs in the lowland forests, but it also has been collected from montane forest in Yaibos, Western Highlands Province, Papua New Guinea, at an altitude of $2100 \mathrm{~m}$.

2 . This species is uniquely characterized by its few and strongly arcuate ascending secondary veins, that do not join to form a submarginal vein.

\section{Ochrosia glomerata (Blume) F. Muell.}

Ochrosia glomerata (Blume) F. Muell. (1871) 130; Valeton (1895) 233; Markgr. (1927) 191; Merr. \& L.M. Perry (1943) 213; Pichon (1947) 209; H. St. John (1973) 280. - Pseudochrosia glomerata Blume (1850) 158; K. Schum. (1888) 214; (1895) 156. - Neisosperma glomerata (Blume) Fosberg \& Sachet in Fosberg, Boiteau \& Sachet (1977) 29; Markgr. (1979) 246. - Lactaria glomerata (Blume) Koidz. (1923) 51. - Type: Zippelius s.n. (holo L), Indonesia, Papua.

Tree, 4-30 m high. Branchlets angular to subangular, 3.5-6 mm diam., often sulcate when dried, not lenticellate, glabrous, smooth, with conspicuous leaf scars. Leaves (2or) 3- or 4-whorled, coriaceous when dried, petiolate; petiole $0.6-5.3 \mathrm{~cm}$ long, glabrous; blades (narrowly) obovate or (narrowly) elliptic, $5.4-26$ by $2.1-8 \mathrm{~cm}$, ratio $2.1-5.8$, base decurrent into petiole, margin entire to slightly undulate, apex usually acuminate, sometimes acute, or very rarely shallowly retuse, secondary veins conspicuous, slightly prominent on abaxial side, $28-45$ pairs, $0.1-0.7 \mathrm{~cm}$ spaced, at an angle of $80-90^{\circ}$ to the midrib, straight to rather arcuate ascending near the margin, not reaching the margin, 
joining, forming a submarginal vein; tertiary veins often indistinct. Inflorescence 1.5-18 $\mathrm{cm}$ long, terminal and axillary cymes, in whorls of $2-4$, sometimes solitary, congested, glabrous, sometimes with bracts, pedunculate; peduncle $1-8 \mathrm{~cm}$ long, glabrous, often thick. Flowers fragrant, more than 30 in each inflorescence, pedicellate; pedicels $2 \mathrm{~mm}$ long, glabrous, bracteolate; bracteoles sepal-like, relatively small, ovate, $0.6-0.8$ by $0.6 \mathrm{~mm}$, margin entire, apex rounded, rough outside, smooth inside, glabrous on both sides, 2 or 3 on each pedicel. Sepals ovate, unequal in size, $1.5-2$ by $1.3-2 \mathrm{~mm}$, ratio 1-1.1, margin entire, apex acute, thick except along the margin, glabrous on both sides, rough outside, smooth inside, connate at base for $0.4 \mathrm{~mm}$. Corolla white, in mature buds narrowly ellipsoid, 9 by $2-3 \mathrm{~mm}$, ratio $3-4.5$, of which the lobes form a rounded apex, glabrous outside, villose inside, rather dense, forming a belt of $1.5 \mathrm{~mm}$ wide just below the mouth; tube cylindrical, $3 \mathrm{~mm}$ long, straight, widening at the stamens insertion, and there $2 \mathrm{~mm}$ wide, tube-calyx ratio $1.5-2$, tube-lobes ratio 0.4 ; lobes narrowly elliptic, 8 by $2 \mathrm{~mm}$, ratio 4 , margin entire, apex rounded, glabrous, auriculate on the left side at base. Stamens inserted at $1 \mathrm{~mm}$ below the mouth, 0.7 of the length of the corolla tube; filaments filiform, $0.4 \mathrm{~mm}$ long; anthers ovate to narrowly so, $1.5-1.8$ by $0.5 \mathrm{~mm}$, sagittate at base, apex acute, yellowish when dried. Pistil glabrous, 2.5 $\mathrm{mm}$ long; ovary subglobose, 1 by $1 \mathrm{~mm}$, of 2 distinctly separated carpels, widening at base, apex rounded and abruptly narrowed at base of style; style filiform, $1 \mathrm{~mm}$ long, style-ovary ratio 1 , not split at base; pistil head ovoid or conical, 0.5 by $0.2-0.3 \mathrm{~mm}$. Fruits composed of 2 separate mericarps; mericarps blackish brown or brown when dried, ovoid, ellipsoid, or subglobose, $4.1-7.6$ by $1.8-3.5$ by $1.7-3.5 \mathrm{~cm}$, base rounded to blunt, apex apiculate, acumen often strongly curled, verrucose when dried, glabrous, rather glossy, indehiscent, green and turning yellow or orange when ripe; endocarps split into fibres penetrating the mesocarps; fibres rather thin and slender, not forming hard thick pointed ends. Seeds 2 at either placenta, flat, suborbicular to ellipsoid, 1.3-3 by $1-2.1 \mathrm{~cm}$, apex acuminate, glabrous, whitish.

Distribution - In Malesia: Malaysia (Borneo: Sabah), Philippines (Luzon, Palawan, Mindanao, Sibuyan, Mindoro, Negros) and Indonesia (North Sulawesi, Moluccas, West Papua). Also found in Solomon Islands.

Habitat \& Ecology - In primary and secondary rain forests, closed broadleaved rain forests, riversides, beach forests, swampy areas, disturbed forests or open areas. Also found in forests dominated by Dipterocarpus species, dry forests with Casuarina species or open forests with rattans and climbing bamboos. On red lateritic clay over ultra basic rocks, clay loam, stony soil, or black soil. Altitude 2-900 m.

Note - This species is characterized by its short corolla tube and verrucose fruits (when dried), which make it most similar to $O$. oppositifolia. The shape and size of the leaf blades is very variable.

\section{Ochrosia oppositifolia (Lam.) K. Schum.}

Ochrosia oppositifolia (Lam.) K. Schum. (1895) 156; K. Schum. \& Lauterb. (1901) 504; Merr. (1923) 330; Pichon (1947) 211; Guillaumin (1948) 293; Boiteau, L. Allorge \& Sevenet (1972) 629; H. St. John (1973) 280; Leeuwenb. (1987) 51; D.J. Middleton (1999) 65. - Cerbera oppositifolia Lam. (1783) 62. - Neisosperma oppositifolia Fosberg \& Sachet (1972) 48 (as 'Neiosperma'); Fosberg, Boiteau \& Sachet (1977) 30; Markgr. (1979) 243; Boiteau \& L. Allorge (1981) 66. - Lactaria oppositifolia (Lam.) Kuntze (1891) 415. - Calpicarpum oppositifolium (Lam.) Boiteau in Boiteau et al. (1974) 495; Boiteau, L. Allorge \& Sevenet (1975) 153, nom. illeg. 
- Cerbera salutaris Lour. (1790) 136; Spreng. (1825) 642, superfl. name. - Lactaria salubris Rumph. ex Raf. (1838) 162; Hassk. \& de Vriese (1856) 9; Miq. (1862) 553; Koidz. (1923) 51, superfl. name. - Ochrosia salubris (Raf.) Blume (1850) 158; Miq. (1869) 137; Valeton (1895) 226, superfl. name. - Bleekeria salubris (Raf.) Hassk. (1855) 41, superfl. name. - Calpicarpum lamarckii G. Don (1838) 100, nom. illeg. - Kopsia lamarckii B.D. Jacks. (1895) 12, misapplied name - Type: Rumphius, Herb. Amboin. 2 (1742) 255, t. 84.

Cerbera platyspermos Gaertn. (1791) 193. - Ochrosia platyspermos (Gaertn.) A.DC. (1844) 356.

- Ochrosion platyspermum (Gaertn.) St.-Lag. (1880) 131, nom. nud.

Neisosperma muricata Raf. (1838) 162, nom. illeg.

Ochrosia cowleyi F.M. Bailey (1897) 229; (1900) 982, t. 91/92, f. 5.

Ochrosia elliptica auct. non Labill.: K. Schum. \& Hollrung (1889) 112.

Ochrosia borbonica auct. non J.F. Gmel.: Trimen (1895) 129, pl. 1x.

Ochrosia parviflora sensu Markgr. (1926) 283.

Tree, 2.5-45(-60) $\mathrm{m}$ high. Branchlets terete, often angular towards the upper part of the branchlets, hollow, thick, (4-)6-9 mm diam., often sulcate when dried, not lenticellate, glabrous, with conspicuous leaf scars. Leaves 3- or 4- (or 5-) whorled, coriaceous or papyraceous when dried, long petiolate; petiole 1.5-6.6 cm long, glabrous; blades usually obovate, very rarely elliptic, $6.5-36$ by $3.4-18 \mathrm{~cm}$, ratio $1-3$, base decurrent into petiole, margin entire, apex often rounded or acuminate, sometimes retuse, shallowly retuse, or truncate; secondary veins conspicuous on both sides, not prominent, 21-42 pairs, $0.2-1.5 \mathrm{~cm}$ spaced, at an angle of $70-90^{\circ}$ to the midrib, straight to rather arcuate ascending, not reaching the margin, joining, forming a distinct submarginal vein which is close to the margin; tertiary veins rather inconspicuous. Inflorescence 2-17 cm long, terminal and axillary cymes, in whorls of 2-4, congested, glabrous, sometimes with bracts, pedunculate; peduncle 1-10.5 cm long, glabrous. Flowers fragrant, more than 30 in each inflorescence, fairly long pedicellate; pedicels $2.1-4 \mathrm{~mm}$ long, glabrous, bracteolate; bracteoles sepal-like, ovate, $0.8-1.3$ by $0.6-1 \mathrm{~mm}$, margin entire, apex rounded, rough outside, smooth inside, thick, glabrous on both sides, 2 or 3 on each pedicel. Sepals ovate, $2-2.3$ by $1.8-2 \mathrm{~mm}$, ratio $1-1.2$, margin entire, apex rounded, thick except along the margin, glabrous on both sides, rough outside, smooth inside, connate at base for $1 \mathrm{~mm}$. Corolla white, creamy white, or yellowish white, in mature buds narrowly ellipsoid, $11-15$ by $2-2.5 \mathrm{~mm}$, ratio $4.4-6$, of which the lobes form a rounded apex, glabrous outside, villose inside, rather dense, forming a belt of 1.5-2 mm wide just below the insertion of stamens; tube cylindrical, 5.3-7 mm long, straight, widening at the stamens insertion, and there $2-2.3 \mathrm{~mm}$ wide, tube-calyx ratio $2.5-3.5$, tube-lobes ratio $0.5-0.7$; lobes narrowly elliptic, $9-12$ by $3.5-4 \mathrm{~mm}$, ratio 2.6-3, margin entire, apex rounded, glabrous, auriculate on the left side at base. Stamens inserted at $2-2.5 \mathrm{~mm}$ below the mouth, $0.5-0.7$ of the length of the corolla tube; filaments filiform, $0.5-0.6 \mathrm{~mm}$ long; anthers ovate to narrowly so, $1.2-1.5$ by $0.5-0.8$ $\mathrm{mm}$, sagittate at base, apex acute, yellowish when dried. Pistil glabrous, 2.5-3.5 mm long; ovary subglobose, widening at base, apex rounded and abruptly narrowed at base of style, 1 by $1 \mathrm{~mm}$, of 2 distinctly separated carpels; style filiform, 1-1.9 mm long, style-ovary ratio $1-1.9$, thick, not split at base; pistil head ovoid or conical, $0.5-0.6$ by $0.3 \mathrm{~mm}$. Fruits composed of 2 separate mericarps; mericarps blackish brown or light brown when dried, ovoid to ellipsoid, sometimes subglobose, $5-8.5$ by $3-5.1$ by $2.8-4$ $\mathrm{cm}$, apex apiculate, acumen strongly curled, sometimes acuminate, remotely verrucose when dried, glabrous, indehiscent, green and turning yellow or orange when ripe; endocarps split into fibres penetrating the mesocarps; fibres hard and thick or rather thin 
and slender, forming hard thick pointed ends or not. Seeds often 1 at either placenta, flat, suborbicular to ellipsoid, $2.6-3.8$ by $1.9-2.9 \mathrm{~cm}$, rarely with a small second one, flat, ellipsoid, 1-2.5 by $0.7-1.7 \mathrm{~cm}$, apex acuminate, glabrous, brown.

Distribution - Sri Lanka, Andaman Islands, Thailand. In Malesia: Malaysia (Peninsular Malaysia, Borneo: Sabah, Sarawak), Philippines (Mindanao, Polilo, Luzon, Mindoro, Babuyanes), Indonesia (Sumatra, Java, South Sulawesi, Moluccas, West Papua), and Papua New Guinea. Widespread throughout Pacific Islands: Samoa, Marshal Islands, Fiji, Vanuatu, Mariana Islands, Solomon Islands, Tonga, Caroline Islands, New Caledonia, Seychelles Islands, Tahiti, and Austral Islands.

Habitat \& Ecology - Rain forests by seashore, beach vegetation, disturbed primary forests on deep coral sand, edge of mangrove swamps, or open areas. On coral sand and gravel. Altitude $0-200 \mathrm{~m}$.

Notes -1 . This species is characterized by its large leaf blades and short corolla tubes. The mericarps look like those of $O$. glomerata, but less (remotely) verrucose. They are also different in the number of seeds (often 1 or rarely with a second small one at either placenta in $O$. oppositifolia vs. always 2 in $O$. glomerata).

2. This is the most collected Ochrosia species.

3. Mostly found at very low altitudes, from 0 to less than $100 \mathrm{~m}$ asl. Only a few specimens have been collected from an altitude of 175-400 $\mathrm{m}$ (in Tinian Islands and Rota in Mariana Islands, and Malili in South Sulawesi).

4. The genus Calpicarpum G. Don was reduced to Kopsia Blume by Alphonse de Candolle in 1844 by excluding C. lamarckii G. Don (= Cerbera oppositifolia Lam. = Ochrosia oppositifolia (Lam.) K. Schum.). So the name Kopsia lamarckii is a misapplied name.

\section{Ochrosia sciadophylla Markgr.}

Ochrosia sciadophylla Markgr. (1967) 26. - Neisosperma sciadophylla (Markgr.) Fosberg \& Sachet in Fosberg, Boiteau \& Sachet (1977) 31; Markgr. (1979) 246. - Type: Whitmore's collectors BSIP 3700 (holo BSIP n.v.; iso L), Solomon Islands, New Georgia Islands, Vaimbu.

Tree, 9-36 m high. Branchlets angular, 4-5 mm diam., sulcate when dried, not lenticellate, glabrous, smooth, leaf scars often conspicuous, blackish. Leaves 4-whorled, coriaceous or thinly so when dried; petiole $2-4.7 \mathrm{~cm}$ long, thin, glabrous; blades narrowly elliptic, $18.1-24$ by $4.8-5.7 \mathrm{~cm}$, ratio $3.2-4.5$, base cuneate, margin entire or slightly undulate, apex usually acuminate, sometimes acute; secondary veins conspicuous, prominent on abaxial side, 20-22 pairs, 0.6-1.4 cm spaced, at an angle of $80-85^{\circ}$ to the midrib, arcuate ascending, not reaching the margin, joining, forming a submarginal vein, which is close to the margin; tertiary veins not prominent, usually conspicuous only on abaxial side, reticulate. Inflorescence $3-6.5 \mathrm{~cm}$ long, terminal and axillary cymes, in whorls of 3 or 4 , loose, glabrous, sometimes with bracts, pedunculate; peduncle $0.7-4.5 \mathrm{~cm}$ long, glabrous. Flowers fragrant, 10-12 in each inflorescence, pedicellate; pedicels $3 \mathrm{~mm}$ long, glabrous, bracteolate; bracteoles sepal-like, ovate, 1 by $1 \mathrm{~mm}$, margin entire, apex acute, thick, rough outside, smooth inside, glabrous, 1 or 2 on each pedicel. Corolla white or sometimes yellowish white. Fruits composed of 2 separate mericarps; mericarps blackish brown when dried, ovoid, 4.9-6.5 by $2.5-3.4$ by $2.3-3.1 \mathrm{~cm}$, base rounded, apex usually apiculate, acumen $0.5-0.8 \mathrm{~cm}$ 
long, usually strongly curled at the apical part, verrucose when dried, glabrous, smooth, rather glossy, indehiscent, green when unripe; endocarps split into fibres penetrating the mesocarps; fibres rather thin and slender, not forming hard thick pointed ends. Seeds 1 at either placenta, flat, ellipsoid, 2.9-3.4 by $1.5-1.8 \mathrm{~cm}$, base rounded, apex cuspidate, glabrous, whitish brown.

Distribution - Malesia: Papua New Guinea. Mostly found in the Solomon Islands.

Habitat \& Ecology - In primary and secondary lowland rain forests. On volcanic rock. Altitude 9-170 m.

Notes -1 . Specimens with mature buds and open flowers are not available.

2 . The secondary veins are arcuate ascending like those of $O$. ficifolia but it has many-nerved leaves (which are less than 15 pairs in $O$. ficifolia), thin and slender fibres (which are thick and hard in $O$. ficifolia) and one seed at either placenta (which are 3 in O.ficifolia).

\section{OCHROSIA section OCHROSIA}

Ochrosia Juss. sect. Ochrosia. - Ochrosia Juss. sect. Lactaria F. Muell. (1871) 130; Pichon (1947) 206. - Ochrosia Juss. subg. Lactaria (F. Muell.) Valeton (1895) 225. - Ochrosia sensu Fosberg \& Sachet (1972) 47; (1974) 254; Fosberg, Boiteau \& Sachet (1977) 23; Markgr. (1979) 233; Boiteau \& L. Allorge (1981) 47. - Type: Ochrosia borbonica J.F. Gmel.

Bleekeria Hassk. emend. Koidz. (1923) 51. - Type: Bleekeria kalokarpa Hassk. (= Ochrosia elliptica Labill.).

Excavatia Markgr. (1927) 194; (1936) 127. — Lectotype: Excavatia littoralis (Merr.) Markgr. (= Ochrosia ackeringae (Teijsm. \& Binn.) Miq.), designated by Leeuwenberg, 1987.

Fruits apocarpous, rarely syncarpous or hemisyncarpous. Mericarps often smooth when dried, often dorsoventrally compressed or biconvex in transverse section. Endocarps hard, thick, massive, surrounding 2 lateral cavities.

\section{KEY TO THE SPECIES OF OCHROSIA SECTION OCHROSIA}

1a. Peduncle of inflorescences not winged. Calyx ciliolate at margin. Fruits syncarpous

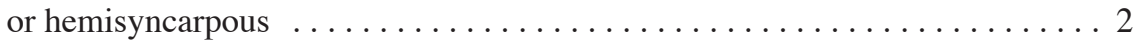

b. Peduncle of inflorescences winged (unknown in $O$. tenimberensis). Calyx not ciliolate at margin (unknown in O. tenimberensis). Fruits apocarpous . . . . . 3

2a. Branchlets terete, 1-2 mm diameter. Leaf blades ratio 4.2-7.1, secondary veins at an angle of $45-50^{\circ}$ to the midrib. Fruits syncarpous . . . . 11. O. syncarpa

b. Branchlets subangular to angular upwards, $2-4 \mathrm{~mm}$ diameter. Leaf blades ratio $2.1-4.6$, secondary veins at an angle of $75-85^{\circ}$ to the midrib. Fruits hemisyncarpous $\ldots \ldots \ldots \ldots \ldots \ldots \ldots \ldots \ldots \ldots \ldots \ldots \ldots \ldots \ldots \ldots \ldots$ 8. ackeringae

3a. Branchlets rather thick, not slender, more than $2 \mathrm{~mm}$ diameter. Corolla tube more than $12 \mathrm{~mm}$ long (unknown in O. tenimberensis). Mericarps $4 \mathrm{~cm}$ long or more

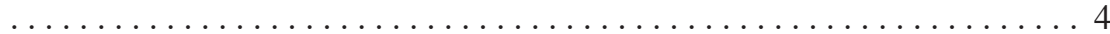

b. Branchlets thin, slender, 1-1.5 mm diameter. Corolla tube $4 \mathrm{~mm}$ long or less.

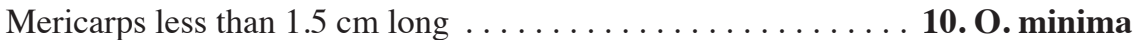

4a. Leaves papyraceous when dried, apex cuspidate. Mericarps with distinct lateral ridges, strongly dorsoventrally compressed. Seeds 2 at either placenta . . . . . . 
b. Leaves coriaceous when dried, apex acute to acuminate. Mericarps without lateral ridges, not compressed dorsoventrally. Seeds 3 at either placenta . . . . . . . . .

12. O. tenimberensis

\section{Ochrosia ackeringae (Teijsm. \& Binn.) Miq.}

Ochrosia ackeringae (Teijsm. \& Binn.) Miq. (1869) 138; F. Muell. (1871) 131; Valeton (1895) 229; Pichon (1947) 208; Fosberg, Boiteau \& Sachet (1977) 23; Markgr. (1979) 238. - Lactaria ackeringae Teijsm. \& Binn. (1867) 249. - Bleekeria ackeringae (Teijsm. \& Binn.) Koidz. (1923) 52. - Type: Ackeringa s.n. (holo L), Indonesia, Sumatra, Bangka.

Ochrosia ackeringae (Teijsm. \& Binn.) Miq. var. angustifolia Baker f. (1900) 182. - Type: not indicated, but the description given by the author refers to $O$. ackeringae.

Ochrosia littoralis Merr. (1909) 315; Merr. (1923) 330; Pichon (1947) 208. - Bleekeria littoralis (Merr.) Koidz. (1923) 52. - Excavatia litoralis (Merr.) Markgr. (1927) 194. - Type: Curran \& Merrtit FB 7754 (holo PNH $\dagger$ ), Philippines, Luzon, Batangas, Malabrigo. - I have not found any specimen of Curran \& Merritt FB 7754, but the description given in the protologue ("Drupes united at the base, divaricate ...") refers to $O$. ackeringae.

Lactaria calocarpa auct. non Hassk.: Miq. (1862) 553. - Refers to the Teijsmann's specimen from Poeloe Seboekoe, Sumatra (see Miq. (1869) 138). The description given matches the characters of $O$. ackeringae.

Shrub or tree, 3-10 m high. Branchlets terete, usually angular to subangular towards the upper part of the branchlets, thin, 2-4 mm diam., sometimes sulcate, smooth, not lenticellate, glabrous, often blackish when dried. Leaves 2- or 3-whorled, coriaceous or papyraceous when dried; petiole $0.4-1.6 \mathrm{~cm}$ long, glabrous; blades obovate, elliptic, or narrowly so, $5.5-20$ by $2.5-4.4 \mathrm{~cm}$, ratio $2.1-4.6$, base decurrent onto petiole, or cuneate, margin entire, apex usually acuminate, sometimes acute or cuspidate, very rarely shallowly retuse, rounded, or truncate; secondary veins inconspicuous, not prominent, more than 35 pairs, $0.1-0.2 \mathrm{~cm}$ spaced, at an angle of $75-85^{\circ}$ to the midrib, straight to rather arcuate ascending near the margin, not reaching the margin, joining, forming a submarginal vein; tertiary veins indistinct. Inflorescence 2.5-10.7 $\mathrm{cm}$ long, terminal and axillary cymes, in whorls of 2 or 3 , repeatedly dichotomous, loose or congested, glabrous, sometimes with bracts, pedunculate; peduncle 1-6.9 cm long, glabrous. Flowers fragrant, 8-20 on each inflorescence; pedicels 2-2.5 mm long, glabrous, bracteolate; bracteoles sepal-like, broadly ovate, $1.1-1.2$ by $1.3-1.4 \mathrm{~mm}$, margin entire, apex rounded, rather thin, rough outside, smooth inside, glabrous, 2 or 3 on each pedicel. Sepals ovate to broadly so, $1.9-2.3$ by $1.5-2 \mathrm{~mm}$, ratio $0.9-1.5$, margin ciliolate, apex rounded, middle part of the lower half thick, glabrous, ciliolate outside, smooth inside, connate at base for $0.7 \mathrm{~mm}$. Corolla white, in mature buds narrowly ellipsoid, 18 by $2 \mathrm{~mm}$, ratio 9, of which the lobes form a rounded apex, glabrous outside, villose inside, rather dense, forming a belt of $2 \mathrm{~mm}$ wide just below the insertion of stamens; tube cylindrical, $12.5 \mathrm{~mm}$ long, straight, widening at stamens insertion, and there $2 \mathrm{~mm}$ wide, tube-calyx ratio 5.4-6.6, tube-lobes ratio 1; lobes narrowly ovate, 12 by $3 \mathrm{~mm}$, ratio 4 , margin entire, apex rounded or acute, glabrous, auriculate on the left side at base. Stamens inserted at $3 \mathrm{~mm}$ below the mouth, 0.8 of the length of the corolla tube; filaments filiform, $0.5 \mathrm{~mm}$ long; anthers ovate, 1.3 by $0.5 \mathrm{~mm}$, sagittate at base, apex acute, yellowish when dried. Pistil glabrous, 9 mm long; ovary ovoid, 1.9 by $1 \mathrm{~mm}$, of 2 distinctly separated carpels, widening at base, gradually narrowed at apex towards base of style; style filiform, $6.5 \mathrm{~mm}$ long, style-ovary ratio 4-5, split at base; pistil head ovoid, 0.5 by $0.3 \mathrm{~mm}$. Fruits hemisyncarpous, V-shaped; mericarps united 
at base for $0.5-2.2(-3.2) \mathrm{cm}$, unequal in size, sometimes only one carpel developing, brown when dried; each mericarp ellipsoid or narrowly so, sometimes narrowly obovoid, $2-4.4$ by $0.6-1.9$ by $0.5-2 \mathrm{~cm}$, base narrowed or rounded, apex acute or acuminate, acumen short and minute, smooth, glabrous, indehiscent, green and turning red when ripe; endocarps massive, hard, surrounding 2 lateral spongy cavities of $0.3-0.4 \mathrm{~cm}$ diameter. Seeds 2 or 3 at either placenta, flat, suborbicular to ellipsoid, $0.4-0.9$ by 0.3-0.6 cm, apex acuminate, glabrous, whitish brown.

Distribution - In Malesia: Malaysia (Borneo: Sabah), Philippines (Luzon, Palawan, Samar, Babuyanes, Mindanao), Indonesia (Sumatra, Java, Sulawesi, Moluccas), Papua New Guinea. Also found in Australia (Christmas Island) and the Solomon Islands.

Habitat \& Ecology - Primary forests behind the beach, upper basin, along the riverbanks, or open areas. On sandy soil, or shallow phosphatised limestone scree and brown clay loam. Altitude $0-300 \mathrm{~m}$ (mostly found at very low altitudes).

Note - Uniquely characterized by its hemisyncarpous fruits.

\section{Ochrosia coccinea (Teijsm. \& Binn.) Miq.}

Ochrosia coccinea (Teijsm. \& Binn.) Miq. (1869) 138; F. Muell. (1871) 131; Valeton (1895) 230; Pichon (1947) 208; Fosberg, Boiteau \& Sachet (1977) 25; Markgr. (1979) 239. - Lactaria coccinea Teijsm. \& Binn. (1867) 249. - Bleekeria coccinea (Teijsm. \& Binn.) Koidz. (1923) 52. - Excavatia coccinea (Teijsm. \& Binn.) Markgr. (1927) 195. - Type: Von Rosenberg s.n. (holo n.v.), Indonesia, Moluccas, Seram.

Shrub or tree, 2-10 m high. Branchlets terete, angular towards the upper part of the branchlets, 2-2.5 mm diam., not lenticellate, glabrous, smooth, the topmost branchlets rarely winged; wing occurs longitudinally on opposite sides along the length of the branchlets, leaf-like, asymmetric, 0.2-1 mm wide, greenish, glabrous. Leaves 2-4whorled, papyraceous when dried; petiole $0.3-0.7 \mathrm{~cm}$ long, thin, glabrous; blades obovate, sometimes elliptic, to narrowly so, $7-13.3$ by $2.5-5.3 \mathrm{~cm}$, ratio $1.7-3.8$, base cuneate, margin entire to undulate, apex cuspidate; secondary veins conspicuous, rather prominent, straight around the base, rather arcuate ascending near the margin towards the apex, 25-38 pairs, $0.1-0.3 \mathrm{~cm}$ spaced, at an angle of $75^{\circ}$ (around the upper half of the blade) to $90^{\circ}$ (around the lower half of the blade) to the midrib, not reaching the margin, joining, forming a submarginal vein, tertiary veins not prominent, usually inconspicuous. Inflorescence $3.5-5.5 \mathrm{~cm}$ long, axillary cymes, solitary or in whorls of 2 or 3, glabrous, with bracts; peduncle $0.6-2(-3.3) \mathrm{cm}$ long, glabrous, winged; wing occurs longitudinally on opposite sides along the length of the peduncle, leaflike, asymmetric, 0.2-0.8 mm wide, greenish, glabrous. Flowers pedicellate; pedicels 1.3-1.5 mm long, glabrous, bracteolate; bracteoles sepal-like, ovate, 1 by $1 \mathrm{~mm}$, apex rounded to acute, smooth inside, glabrous, 2 or 3 on each pedicel. Sepals ovate, 2 by 1 $\mathrm{mm}$, ratio 2, margin entire, apex rounded, rather thin, glabrous, smooth inside, connate at base for $0.4 \mathrm{~mm}$. Corolla yellow or white, in mature buds narrowly ellipsoid, 13.5 by $2 \mathrm{~mm}$, ratio 6.7, of which the lobes form a rounded apex, glabrous outside, villose inside, rather dense, forming a belt of $2 \mathrm{~mm}$ wide just below the insertion of stamens; tube cylindrical, $13 \mathrm{~mm}$ long, straight, widening at stamens insertion, and there $2 \mathrm{~mm}$ wide, narrower towards the base, tube-calyx ratio 6.5, tube-lobes ratio 1.6; lobes elliptic, 8 by $2.5-3 \mathrm{~mm}$, ratio $2.7-3.2$, margin entire, apex rounded, glabrous, auriculate on the left side at base. Stamens inserted at $4 \mathrm{~mm}$ below the mouth, 0.7 of the length of the 
corolla tube; filaments filiform, $0.8 \mathrm{~mm}$ long; anthers ovate to narrowly so, 1.5 by 0.5 $\mathrm{mm}$, sagittate at base, apex acute, yellowish when dried. Pistil glabrous, $8.5 \mathrm{~mm}$ long; ovary ovoid, 1.5 by $0.8 \mathrm{~mm}$, widening at base, gradually narrowed at apex towards base of style, of 2 distinctly separated carpels; style filiform, $6.5 \mathrm{~mm}$ long, style-ovary ratio 4.3, split at base; pistil head ovoid, $0.5 \mathrm{~mm}$ long. Fruits apocarpous, composed of 2 separate mericarps; mericarps brownish when dried, boat-shape, 4-4.6 by 2-2.9 by $1-1.1 \mathrm{~cm}$, strongly dorsoventrally compressed, base long cuneate, apex rounded and sometimes abruptly acuminate, acumen tiny, with distinct apical and lateral ridges, longitudinally shallowly grooved along the middle on both dorsal and ventral surfaces, smooth, glabrous, slightly glossy, indehiscent, green turning bright red or sometimes crimson when ripe; endocarps massive, thick, hard, surrounding 2 spongy cavities of 0.5-0.9 cm diameter. Seeds 2 at either placenta, flat, ellipsoid, unequal in size, 1-1.7 by $0.5-0.8 \mathrm{~cm}$, apex acute, glabrous, brownish.

Distribution - In Malesia: Indonesia (Moluccas and West Papua), and Papua New Guinea (East Sepik, Morobe).

Habitat \& Ecology - Primary and secondary rain forests. On clayey soil. Altitude $0-1000 \mathrm{~m}$.

Notes -1 . Holotype specimen is not found. It was probably in BO.

2. Specimens with complete inflorescence are not available.

3. Characterized by its papyraceous and cuspidate leaves.

\section{Ochrosia minima (Markgr.) Fosberg \& Boiteau}

Ochrosia minima (Markgr.) Fosberg \& Boiteau in Fosberg, Boiteau \& Sachet (1977) 27; Markgr. (1979) 240; P.I. Forst. (1993) 15. - Excavatia minima Markgr. in Merr. \& L.M. Perry (1940) 199. - Bleekeria minima (Markgr.) Merr. \& L.M. Perry (1943) 214. - Type: Brass 8512 (holo B†; lecto A, designated by Markgraf, 1979; iso BO, BRI, L), Papua New Guinea, Tarara, Wasi Kussa River.

Shrub, $5 \mathrm{~m}$ high. Branchlets terete, thin, slender, 1-1.5 mm diam., not lenticellate, glabrous, blackish when dried. Leaves 2- or 3-whorled, papyraceous when dried, shortly petiolate; petiole $0.6-1 \mathrm{~cm}$ long, glabrous; blades obovate, $5.5-10$ by $3-4.4 \mathrm{~cm}$, ratio $1.8-2.8$, base decurrent onto petiole, margin entire, apex acute or acuminate; secondary veins inconspicuous, not prominent, $20-24$ pairs, $0.2-0.4 \mathrm{~cm}$ spaced, at an angle of $75-85^{\circ}$ to the midrib, straight to rather arcuate ascending near the margin, not reaching the margin, joining, forming a submarginal vein; tertiary veins not prominent, inconspicuous. Inflorescence terminal cymes, glabrous, with bracts; peduncle 3.5-6 cm long, glabrous, winged; wing very narrow, 1-1.5 mm wide, occurs on opposite sides along the length of peduncle, leaf-like, yellowish, glabrous. Flowers pedicellate; pedicels 1-3.5 $\mathrm{mm}$ long, glabrous, bracteolate; bracteoles sepal-like, clasping the pedicels, triangular to broadly so, $1-1.1$ by $0.9-1 \mathrm{~mm}$, margin entire, apex acute or rounded, slightly rough outside, smooth inside, glabrous on both sides, 2 or 3 on each pedicel. Sepals ovate, $1.8-2$ by $0.7-0.9 \mathrm{~mm}$, ratio 2.3 , margin entire, apex rounded, erect, thick except along the margin, glabrous on both sides, rough outside, smooth inside, connate at base for $1 \mathrm{~mm}$. Corolla glabrous outside, villose inside, rather dense, forming a belt of $1 \mathrm{~mm}$ wide just around the insertion of stamens; tube cylindrical, 3.9-4 mm long, straight, slightly widening at the stamens insertion, and there $2 \mathrm{~mm}$ wide, tube-calyx ratio $2-2.2$, tube-lobes ratio $1.2-1.3$; lobes elliptic, $3-3.2$ by $1.5 \mathrm{~mm}$, ratio $2-2.1$, margin entire, 
apex rounded, glabrous, auriculate on the left side at base. Stamens inserted at $2-3 \mathrm{~mm}$ below the mouth, 0.5 of the length of the corolla tube; filaments filiform, $0.4-0.5 \mathrm{~mm}$ long; anthers ovate, $1.4-1.5$ by $0.5 \mathrm{~mm}$, sagittate at base, apex acute, yellowish when dried. Pistil glabrous, $3.1 \mathrm{~mm}$ long; ovary subglobose, 1 by $1 \mathrm{~mm}$, widening at base, gradually narrowed at apex towards base of style, of 2 distinctly separated carpels; style filiform, $1.4 \mathrm{~mm}$ long, style-ovary ratio 1.4, thick, split at base; pistil head ovoid, 0.6 by $0.3 \mathrm{~mm}$. Fruits apocarpous, composed of 2 separate small mericarps; mericarps glossy red when fresh, blackish brown or light brown when dried, ellipsoid, 1.2-1.4 by $0.6-1 \mathrm{~cm}$, apiculate at the apex, acumen very short, smooth, glabrous, indehiscent, red when ripe; endocarps massive, hard, surrounding 2 lateral spongy cavities which are occupying the whole space at either side of the seed chamber.

Distribution - Malesia: Papua New Guinea. Also found in Australia (Queensland).

Habitat \& Ecology - In rain forests, or notophyll vineforests on white sandy soil. Altitude $120 \mathrm{~m}$.

Notes -1 . Uniquely characterized by its small mericarps.

2. This species has been very infrequently collected in Malesia. There are a few additional collections from Australia.

3. Specimens with complete inflorescence are not available.

\section{Ochrosia syncarpa Markgr.}

Ochrosia syncarpa Markgr. (1979) 237. - Type: Kostermans 22012 (holo L; iso A, BO), Indonesia, Nusa Tenggara Timur, Flores.

Shrub, $6 \mathrm{~m}$ high. Branchlets terete, thin, 1-2 mm diam., not lenticellate, glabrous, smooth. Leaves 3-whorled, papyraceous when dried, shortly petiolate; petiole $0.4-1$ $\mathrm{cm}$ long, glabrous; blades narrowly elliptic to narrowly obovate, $5-8.3$ by $1-1.7 \mathrm{~cm}$, ratio 4.2-7.1, base decurrent into petiole, margin entire, apex acute; secondary veins inconspicuous, not prominent, straight, $18-30$ pairs, $0.1-0.3 \mathrm{~cm}$ spaced, at an angle of $45-50^{\circ}$ to the midrib, not reaching the margin, joining, forming a submarginal vein; tertiary veins indistinct. Inflorescence $2-3.4 \mathrm{~cm}$ long, terminal and axillary cymes, solitary or in whorls of 2 or 3 , loose, glabrous, sometimes with bracts; peduncle 1-2.1 $\mathrm{cm}$ long, thin, slender, glabrous. Flowers pedicellate; pedicels $2 \mathrm{~mm}$ long, glabrous, bracteolate; bracteoles sepal-like, ovate, 1.1 by $0.8 \mathrm{~mm}$, apex rounded, thick at the middle of basal part, ciliolate outside, smooth inside, glabrous, 1 or 2 on each pedicel. Sepals ovate, unequal in size, $1.8-2$ by $1-1.1 \mathrm{~mm}$, ratio $1.6-1.8$, margin ciliolate, apex rounded, thick at the middle-basal part, thin along the margin, ciliolate outside, smooth inside, glabrous, connate at base for $0.7 \mathrm{~mm}$. Corolla white, in mature buds narrowly ellipsoid, 12 by $2 \mathrm{~mm}$, ratio 6, of which the lobes form a rounded apex, glabrous outside, villose inside, rather dense, forming a belt of $1.5 \mathrm{~mm}$ wide just below the insertion of stamens; tube cylindrical, $10 \mathrm{~mm}$ long, straight, widening at stamens insertion, and there $2 \mathrm{~mm}$ wide, narrower towards the base, tube-calyx ratio 5-5.6, tube-lobes ratio 1.4 ; lobes obovate, 7 by $2.8 \mathrm{~mm}$, ratio 2.5 , margin entire, apex rounded, glabrous. Stamens inserted at $2-2.5 \mathrm{~mm}$ below the mouth, $0.7-0.8$ of the length of the corolla tube; filaments filiform, $0.5 \mathrm{~mm}$ long; anthers ovate to narrowly so, $1-1.1$ by $0.8 \mathrm{~mm}$, sagittate at base, apex acute, yellowish when dried. Pistil glabrous, $5.6 \mathrm{~mm}$ long; ovary ovoid, 1 by $0.7 \mathrm{~mm}$, widening at base, gradually narrowed at apex towards base of style, of 
2 distinctly separated carpels; style filiform, 4.1-6 mm long, style-ovary ratio 4.1-6, split at base; pistil head ovoid, $0.5 \mathrm{~mm}$ long. Fruits syncarpous, blackish brown when dried, ovoid, 1.7-3 by $1-1.5$ by $1.2-1 \mathrm{~cm}$, base rounded, apex acute, smooth, glabrous, slightly glossy, indehiscent, green when unripe; endocarps massive, hard, surrounding 4 spongy cavities of about $0.3 \mathrm{~cm}$ diameter. Seeds 2 at either placenta, flat, ellipsoid, 0.6 by $0.3 \mathrm{~cm}$, apex acuminate, glabrous, whitish.

Distribution - Malesia: Indonesia (Nusa Tenggara).

Habitat \& Ecology - Dry places. Altitude 0-700 m.

Note - Uniquely characterized by its syncarpous fruit.

\section{Ochrosia tenimberensis Markgr.}

Ochrosia tenimberensis Markgr. (1979) 239. - Type: Pleyte 72 (holo L; iso A), Indonesia, Moluccas, Tanimbar Islands, Jamdena, Saumlaki.

Shrub, 3-10 m high. Branchlets terete, angular towards the upper part of the branchlets, 4-5 mm diam., sometimes sulcate when dried, not lenticellate, glabrous. Leaves usually 4-whorled, rarely 3- or 5-whorled, coriaceous when dried, shortly petiolate; petiole $0.3-2.2 \mathrm{~cm}$ long, glabrous; blades elliptic to narrowly so, $8.5-19.5$ by $3.3-6.3$ $\mathrm{cm}$, ratio 2.1-3.3, base decurrent into petiole, margin entire sometimes rather undulate, apex gradually acuminate or acute, secondary veins conspicuous, not prominent, 27-42 pairs, $0.2-0.5 \mathrm{~cm}$ spaced, at an angle of $75^{\circ}$ to the midrib, usually straight, sometimes straight and rather arcuate ascending near the margin, not reaching the margin, joining, forming a submarginal vein, tertiary veins not prominent, conspicuous, reticulate. Flowers white or yellowish white. Fruits apocarpous, composed of 2 separate mericarps; mericarps brown when dried, obovoid to ellipsoid, $4-4.7$ by $1.7-2$ by $1.3-1.7 \mathrm{~cm}$, base narrowed, apex apiculate, acumen $3 \mathrm{~mm}$ long, smooth, glabrous, indehiscent, green and turning red when ripe; endocarps massive, hard, whitish, surrounding 2 spongy cavities of about $0.5 \mathrm{~cm}$ diameter. Seeds 3 at either placenta, flat, ellipsoid, unequal in size, the first two $0.8-1.1$ by $0.6-0.9 \mathrm{~cm}$, the third one 0.7 by $0.3 \mathrm{~cm}$, apex acuminate or rounded, glabrous, whitish.

Distribution - Malesia: Indonesia (Moluccas).

Habitat \& Ecology - Coastal forests or open areas at low altitude.

Notes -1 . Specimens with mature buds, open flowers, and inflorescences are not available.

2. In the protologue of this species, Markgraf designated Pleyte 72 (deposited in L) as the holotype, but the holotype label (with his handwriting on it) was attached on the sheet of the wrong specimen (Pleyte 128, also deposited in L).

\section{SECTION UNKNOWN}

\section{Ochrosia basistamina Hendrian, spec. nov. - Fig. 2}

Ramuli subangulares vel angulares $8-10 \mathrm{~cm}$ diametra. Folia 4- ad 6-vel 7-verticillata, coriacea, petiolus $2-5.5 \mathrm{~cm}$ longus, lamina anguste obovata vel obovata vel elliptica, $14-29 \times 5.5-10.1 \mathrm{~cm}$, venae camptodromae. Inflorescentiae 9.5-18.5 cm longae. Corollae tubus $10 \times 2 \mathrm{~mm}$, lobi elliptici, $12-12.5 \times 3 \mathrm{~mm}$. Stamina in basi tubo insertae. Ovarium $1 \mathrm{~mm}$ altum. Fructus ignotus. - Typus: Boschproefstation 408 (holo L; iso A), Indonesia, South Sulawesi, Malili. 


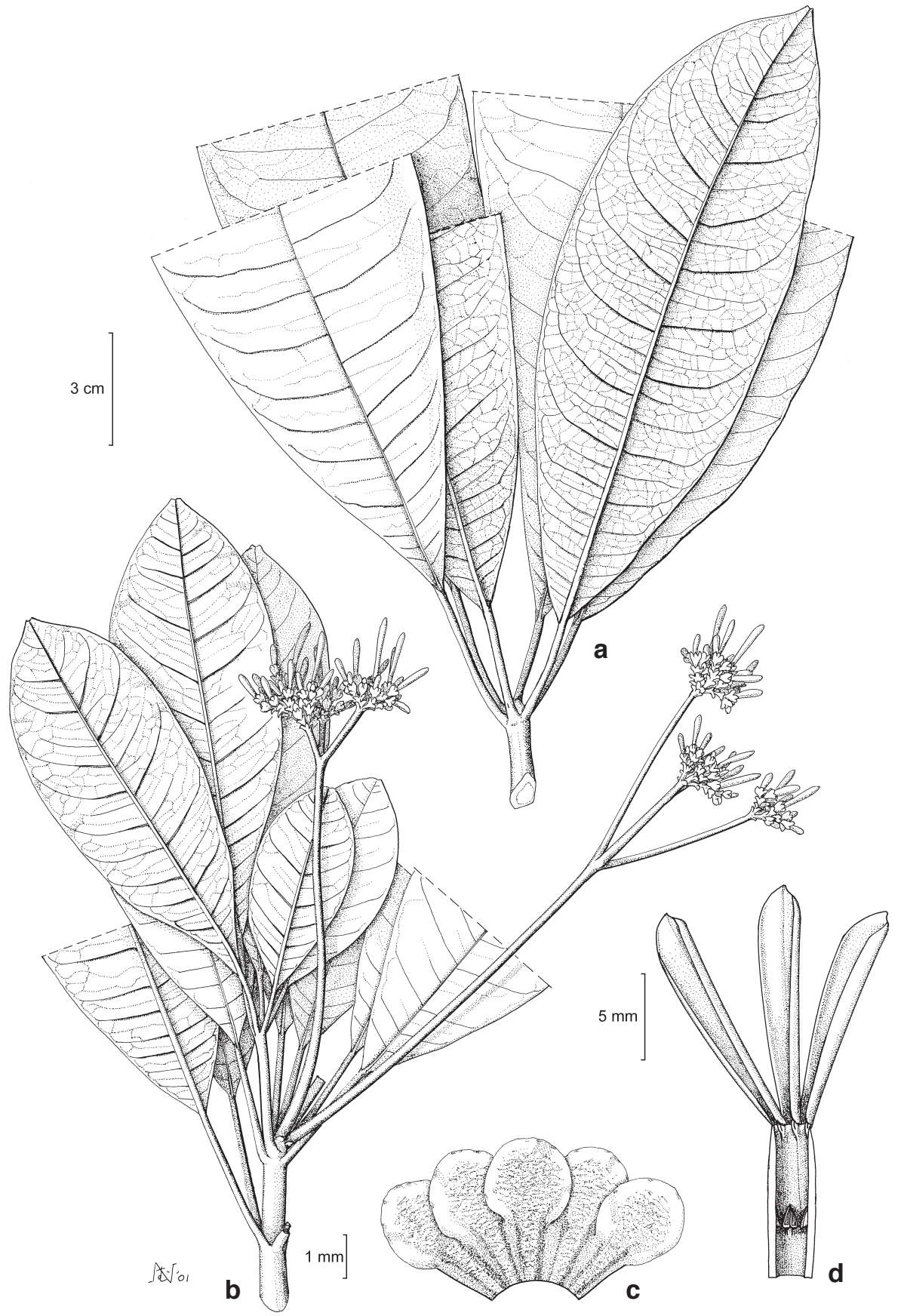

Fig. 2. Ochrosia basistamina Hendrian. a. Leaves; b. leaves and inflorescences; c. sepals; d. stamens insertion (a: Boschproefstation 333, L; b-d: Boschproefstation 408, L). 
Branchlets subangular to angular, thick, $8-10 \mathrm{~mm}$ diam., sulcate when dried, smooth, not lenticellate, glabrous, with conspicuous leaf scars. Leaves 4-6(-7)-whorled, coriaceous to thickly so when dried, long petiolate; petiole $2-5.5 \mathrm{~cm}$ long, glabrous; blades usually narrowly obovate, sometimes obovate or elliptic, $14-29$ by $5.5-10.1 \mathrm{~cm}$, ratio $2-3.1$, base cuneate, margin entire, sometimes rather undulate, apex abruptly acuminate, rarely rounded; secondary veins conspicuous, rather prominent, $12-19$ pairs, $0.5-2.1$ $\mathrm{cm}$ spaced, at an angle of $50-80(-90)^{\circ}$ to the midrib, arcuate ascending, not reaching the margin, usually not joining, rarely joining, not forming a submarginal vein; tertiary veins not prominent, conspicuous on abaxial side only, reticulate. Inflorescence $9.5-18.5 \mathrm{~cm}$ long, terminal and axillary cymes, in whorls of $2-5$, congested, glabrous, long pedunculate; peduncle $6.5-12.5 \mathrm{~cm}$ long, glabrous. Flowers more than 30 on each inflorescence, fairly long pedicellate; pedicels $3 \mathrm{~mm}$ long, glabrous. Sepals suborbicular to orbicular, 2.1 by $2.1 \mathrm{~mm}$, ratio 1, margin entire to undulate, apex blunt, rather thin except at the middle-basal part, rather rough outside, smooth inside, glabrous, connate at base for $0.6 \mathrm{~mm}$. Corolla in mature buds narrowly ellipsoid, 20 by $2-2.5 \mathrm{~mm}$, ratio 8-10, of which the lobes form a rounded apex, glabrous outside, villose inside, sparse, forming a belt of $3 \mathrm{~mm}$ wide just around the insertion of stamens; tube cylindrical, 10 $\mathrm{mm}$ long, straight, tube-calyx ratio 4.8 , tube-lobes ratio 0.8 ; lobes narrowly elliptic, $12-12.5$ by $3 \mathrm{~mm}$, ratio $4-4.2$, margin entire, apex rounded, glabrous, auriculate on the left side at base. Stamens inserted at $8 \mathrm{~mm}$ below the mouth, 0.2 of the length of the corolla tube; filaments filiform, $1 \mathrm{~mm}$ long; anthers ovate to narrowly so, 1.5 by $0.5 \mathrm{~mm}$, sagittate at base, apex acute, yellowish when dried. Ovary ovoid, widening at base, apex rounded and abruptly narrowed at base of style, 1 by $1 \mathrm{~mm}$, of 2 distinctly separated carpels; style filiform, $3.7 \mathrm{~mm}$ long, style-ovary ratio 3.7, not split at base.

Distribution - Malesia: Indonesia (Sulawesi).

Habitat \& Ecology - Altitude $200 \mathrm{~m}$.

Notes -1 . Specimens with fruits are not available. Therefore, the position of this species at the sectional level is still unknown.

2. The species is characterized by the absence of a submarginal vein, its suborbicular to orbicular sepals, and the insertion of stamens near the base (only 0.2 of the length of the corolla tube).

3. It appears to be most similar to O. ficifolia in having arcuate ascending secondary veins and in the absence of a submarginal vein (the submarginal vein is present in other Ochrosia species). But they are different in inflorescence length (which is shorter in O. ficifolia), blade texture (coriaceous to thickly so when dried vs. papyraceous in $O$. ficifolia), the phyllotaxis (usually 5- or 6-whorled vs. 2-4-whorled in O.ficifolia), and sepals (suborbicular to orbicular vs. ovate in $O$. ficifolia).

4. These specimens were all collected from Malili, South Sulawesi. Two of them previously recognized as O. oppositifolia by Markgraf (1979), Leeuwenberg (determination of Boschproefstation 408 of A (1992) and L (1986) specimens), and Fosberg (determination of Boschproefstation 408 of A specimens, 1976). However, in O. oppositifolia the submarginal vein is always present (usually close to the margin, but distinctly recognisable), the secondary veins are many (21 to more than 40 pairs), the corolla tube is much shorter, and the stamen insertion is at about the middle $(0.5-0.7$ of the length of the corolla tube). 
Specimens examined: INDONESIA: Boschproefstation 333 (L), Sulawesi: South Sulawesi, Malili; Boschproefstation 408 (A, L, type), Sulawesi: South Sulawesi, Malili; Neth. Ind. For. Service 220 (A), Sulawesi: South Sulawesi, Malili.

\section{ACKNOWLEDGEMENTS}

This work was enabled by the STUNED Scholarship Programme organised by the Netherlands Education Centre (NEC).

I am most grateful to Prof. Pieter Baas for his invaluable support; and to Dr. Marco Roos, Dr. David J. Middleton and Dr. A.J.M. Leeuwenberg for their supervision.

I would like to thank Dr. Frits Adema and Dr. Peter van Welzen for their help and assistance; and all colleagues at the Nationaal Herberium Nederland, Universiteit Leiden branch, for their help and hospitality. I also wish to thank the directors and curators of the following herbaria whose material has been consulted: A, BM, BO, CANB, GH, K, L, M, NSW, P, UC, WAG, and WRSL. Finally I would like to express my deepest gratitude to Miet van de Wiele, Audrey Selzner and Elena Kamilarova for helping me to translate some articles from French and Dutch to English; and Mr. Jan van Os and Mrs. Anita W. Sachs for the nice drawing.

\section{REFERENCES}

Bailey, F.M. 1897. Contribution to the Flora of Queensland. Queensland Agric. J. 1: 229.

Bailey, F.M. 1900. The Queensland Flora, Part III: 739-1030. Diddams \& Co., Brisbane.

Baker,E.G. 1900. Gamopetalae. In: C.W. Andrews, A monograph of Christmas Island (Indian Ocean): 179-184. British Museum (Natural History), London.

Blume, C.L. 1850. Museum Botanicum Lugduno-Batavum I. Brill, Lugduni-Batavorum.

Boiteau, P. \& L. Allorge. 1981. Flore de la Nouvelle-Calédonie 10. Apocynacées. Muséum National d'Histoire Naturelle, Paris.

Boiteau, P., L. Allorge \& T. Sevenet. 1972. Révision des Ochrosia de Nouvelle-Calédonie. Adansonia sér. 2, 12: 625-629.

Boiteau, P., L. Allorge \& T. Sevenet. 1975. Notes sur les Ochrosiinées de Nouvelle-Calédonie. Adansonia, sér. 2, 15: 147-153.

Boiteau, P., L. Allorge, T. Sevenet \& P. Potier. 1974. Observations morphologiques et chimiotaxonomiques sur les Ochrosiinées de Nouvelle-Calédonie. Adansonia, sér. 2, 14: 485-497.

De Candolle, A. 1844. Apocynaceae. In: A. de Candolle, Prodromus Systematis Naturalis Regni Vegetabilis, Pars VIII: 317-489. Fortin, Masson \& Sociorum, Parisiis.

De Jussieu, A.L. 1789. Genera Plantarum. Herissant \& Barrois, Paris.

De Loureiro, J. 1790. Flora Cochinchinensis 1. Ulyssipone.

Don, G. 1838. A general history of the dichlamydeous plants, vol. IV. Corolliflorae. London.

Elmer, A.D.E. 1912. New Apocynaceae. Leafl. Philipp. Bot. 4: 1445-1468.

Endress, M.E. \& P.V. Bruyns. 2000. A revised classification of the Apocynaceae s.l. Bot. Rev. (Lancaster) 66: 1-56.

Forster, P.I. 1993. A taxonomic revision of Neisosperma Raf. (Apocynaceae) in Australia, together with a key to Australian genera of Apocynaceae. Austrobaileya 4: 13-20.

Fosberg, F.R., P. Boiteau \& M.-H. Sachet. 1977. Nomenclature of the Ochrosiinae (Apocynaceae): 2. Synonymy of Ochrosia Juss. and Neisosperma Raf. Adansonia, sér. 2, 17: 23-33.

Fosberg, F.R. \& M.-H. Sachet. 1972. Plants of southeastern Polynesia. 2. Micronesica 8: 43-49.

Fosberg, F.R. \& M.-H. Sachet. 1974. Plants of southeastern Polynesia. 3. Micronesica 10: 251-256.

Fosberg,F.R. \& M.-H. Sachet. 1977. Nomenclature of the Ochrosiinae (Apocynaceae): 1. Application of the names Neisosperma Raf. and Calpicarpum G. Don. Adansonia, sér. 2, 17: 19-22.

Gaertner, J. 1791. De fructibus et seminus plantarum. Volumen alterum. Tubingae.

Gmelin, J.F. 1791. Systema naturae. Beer, Leipzig.

Greuter, W.J., et al. 2000. International Code of Botanical Nomenclature (Saint Louis Code). Koeltz Scientific Books, Königstein, Germany. 
Guillaumin,A. 1948. Flore analytique et synoptique de la Nouvelle-Calédonie. Phanérogames. Office de la Recherche Scientifique Coloniale, Paris.

Hasskarl, J.C. 1855. Retzia sive observationes botanicae, quas in primis in Horto Botanico Bogoriensi mensibus Februario ad Julium 1855. Lange \& Co., Bataviae.

Hasskarl, J.C. \& W.H. de Vriese. 1856. Eenige kritische onderzoekingen van planten in s'Lands Plantentuin te Buitenzorg. Ned. Kruidk. Arch. 4: 1-13.

Jackson, B.D. 1895. Index Kewensis. Plantarum Phanerogamarum II. Clarendon, Oxford.

Jacquin, N.J. 1791. Collectanea ad Botanicam, Chemiam, et Historiam Naturalem 4. Vindobone.

Koidzumi, G. 1923. Contributiones ad Cognitionem Florae Asiae Orientalis. Bot. Mag. Tokyo 37: $37-59$.

Kuntze, O. 1891. Revisio Genera Plantarum II. Arthur Felix, Leipzig.

Lamarck, M.C. 1783. Encyclopédie Méthodique Botanique 1. Paris.

Leeuwenberg, A.J.M. 1987. The African species of Ochrosia Juss. Series of revisions of Apocynaceae. Part XXIII. Agric. Univ. Wageningen Papers 87, 5: 47-53.

Markgraf, F. 1926. Apocynaceae. Nova Guinea 14, Botanique II: 278-292.

Markgraf, F. 1927. Die Apocynaceen von Neu-Guinea. Bot. Jahrb. Syst. 61: 164-222.

Markgraf, F. 1936. Apocynaceae. In: A.C. Smith, Fijian plant studies. Bernice P. Bishop Mus. Bull. 141: $125-162$.

Markgraf, F. 1967. Notes on the systematy of Solomon Islands' plants and some of their New Guinea relatives, VIII and IX. Gard. Bull. Singapore 22: 23-32.

Markgraf, F. 1979. Florae Malesianae Praecursores LIX. Apocynaceae V. Ochrosia, Neisosperma. Blumea 25: 233-247.

Merrill, E.D. 1909. New or noteworthy Philippine plants, VII. Philipp. J. Sci., Bot. 4: 247-330.

Merrill, E.D. 1923. An enumeration of Philippine flowering plants. Bureau of Science, Manila.

Merrill, E.D. \& L.M. Perry. 1940. Plantae Papuanae Archboldianae, II. J. Arnold Arbor. 21: 163 200.

Merrill, E.D. \& L.M. Perry. 1943. Plantae Papuanae Archboldianae, XII. J. Arnold Arbor. 24: 207217.

Middleton, D.J. 1999. Apocynaceae. In: T. Santisuk \& K. Larsen (eds.), Flora of Thailand 7 : $1-153$.

Miquel, F.A.W. 1862. Sumatra. Van der Post, Amsterdam.

Miquel, F.A.W. 1869. De Quibusdam Rubiaces, Apocyneis et Asclepiadeis. Ann. Mus. Bot. Lugd.Bat. 4: $128-142$.

Moore, S. 1923. Dr. H.O. Forbes' New Guinea plants. Gamopetalae. J. Bot. 61, Suppl.: 23-39.

Mueller, F. 1871. Fragmenta Phytographiae Australiae, Vol. VII. Melbourne.

Pichon, M. 1947. Classification des Apocynacées. III. Genre Ochrosia. Bull. Mus. Natl. d'Hist. Nat., sér. 2, 19: 205-212.

Rafinesque, C.S. 1838. Sylva Tellutiana. Mantissa Synoptica. Philidelphia.

Schumann, K. 1888. Die Flora des Deutschen Ost-Asiatischen Schutzgebietes. Bot. Jahrb. Syst. 9: $186-223$.

Schumann, K. 1895. Apocynaceae. In: A. Engler \& K. Prantl (eds.), Die Natürlichen Pflanzenfamilien, IV, 2: 109-189. Leipzig.

Schumann, K. \& M. Hollrung. 1889. Die Flora von Kaiser Wilhelms Land. Asher \& Co., Berlin.

Schumann, K. \& K. Lauterbach. 1901. Die Flora der Deutschen Schutzgebiete in der Südsee. Leipzig.

Seemann, B. 1865. Flora Vitiensis: A description of the plants of the Viti or Fiji Islands. Reeve \& Co., London.

Sprengel, C. 1825. Systema Vegetabilium, Vol. II, Classis 6-15. Gottingae.

St. John, H. 1973. List and summary of the flowering plants in the Hawaiian Islands. Pacific Tropical Botanical Garden, Memoir Number 1. Lawai, Kauai, Hawaii.

St. Lager, J.B. 1880. Reforme de la Nomenclature Botanique. Ann. Soc. Bot. Lyon 7: 1-154.

Teijsmann, J.E. \& S. Binnendijk. 1867. Plantae Novae. V. Minus Cognitae in Horto Bogoriensi Cultae. Natuurk. Tijdschr. Ned.-Indië 29: 241-259. 
Trimen, H. 1895. A handbook to the Flora of Ceylon. Dulau \& Co., London.

Valeton, T. 1895. Les Ochrosia du Jardin Botanique de Buitenzorg. Ann. Jard. Bot. Buitenzorg 12: $223-237$.

\section{SPECIMENS STUDIED}

Only those specimens with a clearly identified collector and collection number are listed.

Ochrosia section Echinocaryon
1. acuminata
2. apoensis
3. citrodora
4. ficifolia
5. glomerata
6. oppositifolia
7. sciadophylla

Ochrosia section Ochrosia
8. ackeringae
9. coccinea
10. minima
11. syncarpa
12. tenimberensis
Section unknown
13. basistamina

Afaufau 84: 6 - Afriastini 2835: 6 - Ahern et al. FB 2858: 5 - Amin SAN 103171: 6; SAN 126047: 6; SAN 126966: 6 - Anderson 106: 6; 1033: 6; 1202: 6; 2207: 6; 3643: 6 - Asdat 125: 6 - Ashton S 20899: 6.

Backer 28961: 6 - Balakrishnan 3823: 6 - Barbon, Romero \& Fuentes PPI 8151: 2 - Bartlett 15085: 8 - Beaman 9737: 6 - Beer BSIP 7253: 6 - Beer et al. BSIP7831: 5 - Bernardi 13028: 6 - Bhargaya 3320: 6 - Bidin SAN 84736: 6 - Bidin \& Marsak SAN 86145: 6 - Binideh 55154: 5 - Bloembergen bb 29898: 6 - Boschproefstation 29: 1; 64: 8; 333: 13; 351: 1; 408: 13 - Brass 670: 4; 2844: 6; 3442: 5; 8512: 10; 21786: 6 - Bristol 2234: 6; 2454: 6 - BurnMurdoch et al. BSIP 7188: 7 - Buwalda 6095: 6; 7295: 8 .

Carr 12123: 4; 12615: 4; 12616: 4 - Cenabre BS 29970: 8 - Chai SAN 21666: 5 - Chan FRI 37263: 6 - Charoenphol, Larsen \& Warncke 5030: 6 - Cheatam 83: 6 - Christophersen 2635: 6 - Cowmeadow \& Teona BSIP 2529: 7 - Cowmeadow et al. BSIP 3182: 7; BSIP 4727: 7 - Cox 96: 6 - Croft \& Katik NGF 15508: 6 - Croft \& Lelean LAE 65380: 8 - Cuadra A 2442: 5.

Dali JA 7787: 6 - De Bruyn 455: 6 - De Vogel 1386: 6; 2431: 1; 3250: 5; 5214: 1; 5495: 1; 5577: 1; 6110: 1 - De Vogel \& Vermeulen 6546: 1;6677: 1 - Demoulin 5694: 6 - Dewol \& Rahman SAN 89565: 6 - Duenas PNH 34087: 8.

Edaño BS 79330: 6; PNH 17819: 5 - Elbert 3405: 8 - Elmer 10478: 2; 12149: 5; 12783: 5; 14967: 2 - Essig \& Lelean LAE 55050: 6 - Evans 378: 6; 904: 6; 929: 9.

Fa'arodo BSIP 13478: 5 - Falanruw 1883: 6 - Field 38: 6 - Flenley ANU 2298: 4 - Florence 5576: 6; 6184: 6 - Forbes 74: 4; 83: 4 - Foreman \& Katik NGF 48470: 4 - Foreman \& Stocker LAE 60408: 4 - Forman 349: 1 - Forster 8803: 10 - Fosberg 24872: 6; 25114: 6; 25346: 6; 25805: 6; 26145: 6; 26225: 6; 26492: 6; 26735: 6; 26818: 6; 33782: 6; 34038: 6; 34133: 6; 34262: 6; 52067: 6 - Fosberg \& Evans 47105: 6 - Fox PNH 9214: 6 - Frodin UPNG 2175: 6.

Gaerlan, Fuentes \& Romero PPI 5227: 2 - Gaerlan et al. PPI 9915: 5 - Gafui et al. BSIP 8979: 6; BSIP 9252: 6; BSIP 10365: 6; BSIP 10833: 6; BSIP 10989: 6; BSIP 11059: 6; BSIP 14916: 6; BSIP 14976: 6; BSIP 15001: 6; BSIP 15077: 6; BSIP 15239: 5; BSIP 15326: 5; BSIP 16826: 5; BSIP 18330: 6; BSIP 18473: 5; BSIP 18762: 5; BSIP 18871: 6; BSIP 18975: 5 - Gibot 54828: 5 - Gillison NGF 22419: 6; NGF 25292: 6 - Gjellerup 116: 3; 655: 3 - Goetghebeur 3495: 6; 3785: 6 - Guerrero BS 751: 6 - Guppy 337: 8 .

Halligan 1299: 6; 1898: 6 - Hardy 130: 6; 154: 6 - Hartley 9973: 9 - Henderson 331: 6 - Henty NGF 28019: 3 - Henty \& Foreman NGF 42545: 3 - Hoff 3753: 6 - Hollrung 840: 6 - Hoogland \& Craven 10554: 3 - Horgen, Majaducon \& Flores 115: 5 - Horne 504: 6 - Huang, Huang \& Yang 16462: 6 - Hunt 2856: 5.

Isles \& Vinas NGF 34499: 4 - Iwanggin BW 5603: 4; BW 5671: 4; BW 5773: 4; BW 5827: 4. Jacobs 4753: 8; 7721: 5; 7975: 5; 9209: 4 - Jaheri 205: 5 - Jensen 372: 9 - Josiah 53: 6. 
Kajewski 207: 6; 405: 6; 947: 6 - Kalkman BW 3543: 5 - Kanehira \& Hatusima 11424: 4; 13286: 6 - Kanis 1357: 3 - Katik LAE 62246: 3; LAE 64207: 3; NGF 46562: 3; NGF 46663: 3; NGF 46713: 9 - Kerenga et al. LAE 77413: 6 - Kerr 9267: 6; 12671: 6; 18912: 6 - Kjelberg 2587: 1 - Koch 1155: 6 - Koorders \& Valeton 129: 8; 29918: 8 - Koorders 127: 6; 128: 6; 130: 8; 131: 8; 11115: 8; 11116: 8; 15816: 1; 16086: 1; 20360: 8 - Kornassi 861: 6 - Koster BW 301: 4; BW 6749: 4; BW 10958: 4; BW 11185: 3; BW 13523: 4 - Kostermans 172: 4; 284: 9; 387 : 4; 399: 9; 22012: 11 - Kostermans \& Reksodihardjo 23: 3; 272: 3; 360: 3 - Kostermans \& Wirawan 64: 11 .

Lam 3265: 5; 3440: 6 - Lauterbach 1073: 3 - Lee S 41811: 6 - Leeuwenberg, Sidiyasa \& Arbainsyah 14542: 1 - Leonardo 3480: 5 - Liew 225: 6 - Loeters 1662: 11 - Loher 14219: 5.

MacKee 44946: 6 - Maenu' u BSIP 5157: 7; 6110: 6 - Main \& Aden 1480: 8 - Majaducon 8518: 5 - Mann NGF 43232: 6 - Mansur et al. SAN 117472: 5 - Martyn SAN 18406: 8 - Mauriasi et al. BSIP 8623: 6; BSIP 12426: 6; BSIP 13784: 6; BSIP 13881: 5; BSIP 14166: 6; BSIP 14231: 6; BSIP 14513: 6; BSIP 14550: 6; BSIP 15846: 6; BSIP 15994: 6; BSIP 16136: 6; BSIP 16206: 5; BSIP 17073: 6; BSIP 17109: 6; BSIP 17668: 6; BSIP 17750: 6; BSIP 17840: 6; BSIP 17950: 6; BSIP 17957: 6; BSIP 18078: 6; BSIP 18172: 5 - McDonald \& Ismail 3690: 3; 3777 : 3 - McGregor FB 10627: 8 - Meijer 10019: 1 - Mendoza PNH 18397: 2 - Merrill BS 9298: 5 - Millar \& Van den Berg NGF 40904: 6 - Mitchell 34: 8 - Moll BW 9622: 5; BW 15740: 4; BW 15760: 4 - Moran 4611: 6 - Morat 7225: 6.

Necker 164: 6 - NIFS 220: 13; 2629: 6; 21430: 11; 24283: 12; 25643: 3; 25697: 3; 28963: 3; 30273: 4; 30288: 4; bb 17155: 5; bb 17158: 1; bb 17679: 1; bb 22791: 5; bb 24878: 5; bb 31894: 5 .

Orolfo BNB 3797: 8.

Paraiso BS 25492: 5 - Parks 15274: 6; 16368: 6 - Pipoly et al. PPI 38034: 5; PPI 38048: 5; PPI 38295: 5 - Pleyte 72: 12; 128: 12; 472: 4 - Podzorski SMHI 752: 5; SMHI 827: 5; SMHI 2071: 5 - Powell et al. BSIP 19489: 6 - Pullen 1352: 9; 1797: 3 - Putz FRI 23669: 6.

Ramlanto 416: 6 - Ramos BS 13583: 5; BS 23605: 2; BS 27208: 8; BS 27253: 5; BS 41029: 6; FB 1703: 5; FB 1846: 5 - Ramos \& Convocar BS 83968: 5 - Ramos \& Edaño BS 47264: 5 - Raulerson 1512: 6 - Regalado \& Sirikolo 817: 5 - Reillo BS 16284: 6 - Reynoso \& Majaducon PPI 24327: 5 - Ridsdale 1469: 5; NGF 33905: 3; SMHI 178: 5; SMHI 1526: 5; SMHI 1613: 5; SMHI 1713: 5 - Ridsdale, Dejan \& Baquiran ISU 107: 5; ISU 266: 5 - Ridsdale \& Galore NGF 33448: 4 - Ridsdale et al. ISU 494: 5 - Riekerk BW 2392: 5 - Robin 1597: 3 - Rock \& Cooke Jr. 10279: 6 - Rodin 822: 6 - Runikera BSIP 12882: 6 - Runikera et al. BSIP 10639: 5; BSIP 12582: 6; BSIP 12721: 5; BSIP 13189: 7.

Sachet 1773: 6 - Saikeh SAN 68109: 8 - Salvoza BS 29674: 6 - Saunders 437: 3; 1094: 4 - Sayers NGF 19000: 3; NGF 19512: 6; NGF 19533: 3 - Schlechter 17298: 3 - Schmutz 2425: 11 - Schodde 2270: 4 - Schodde \& Craven 4349: 4 - Schram BW 1710: 4; BW 1759: 4; BW 6169: 4; BW 10687: 4; BW 12426: 4 - Schrijn BW 3611: 3 - Seemann 316: 6 - Setchell 15382: 6; 15465: 6 - Sevenet 283: 6 - Shmull 48: 6 - Sinanggul 36023: 5; 57436: 5 - Sinclair 10702: 6 - Singh, Nordin \& Talip 49136: 8 - Sirute'e et al. BSIP 9613: 6 - Smith 1430: 6 - Soejarto \& Madulid 9044: 5 - Soejarto \& Reynoso 6323: 8 - Soejarto et al. 7091:5; 8509: 5 - Sohmer \& Katik LAE 75128: 3 - Sore, Masu'u \& Lipaqeto BSIP 2643: 6 - Spence 386: 6 - St. John 16632: 6 - St. John \& Cowan 21909: 6 - Stokes 49: 6 - Stone, Reynoso \& Fernando PPI 6869: 5 - Streimann LAE 53829: 3 - Streimann \& Kairo NGF 39332: 3 - Streimann \& Lelean LAE 52666: 6 - Sujadi AS 66: 9 - Sulit PNH 12303: 5; PNH 12460: 8; PNH 13833: 8 - Sulit \& Conklin PNH 17709: 5.

Takeuchi 4954: 3 - Talip 47675: 5 - Tamesis FB 20687: 5 - Taylor 46-1091: 6; 46-1107: 6; 461291: 6; 46-1471: 6 - Tecson BS 24682: 6 - Teijsmann 129: 6 - Teona BSIP 6287: 6 - Treub 518: 9.

Van Borssum Waalkes 205: 8; 726: 8; 3059: 6; 3191: 12 - Van Leeuwen 9329: 3 - Van Royen 3508: 5; NGF 16488: 9 - Van Royen \& Sleumer 6454: 3 - Van Slooten 677: 6 - Van Slooten \& Backer 35055: 6 - Verheijen 3394: 11; 3479: 11 - Versteegh BW 3840: 6; BW 4626: 5; BW 4737: 9 - Vinas AV 222: 3 - Vinas, Streimann \& Kairo AV 192: 4 - Vink BW 11364: 4; BW 12100: 9. 
Walker \& White BSIP 199: 7 - Weiblen 502: 6 - White NGF 9537: 9; NGF 10026: 7; NGF 10227: 3 - Whitmore 6346: 6; 6349: 6; BSIP 1344: 6; BSIP 1932: 6; BSIP 1950: 7; BSIP 2482: 6; BSIP 2761: 5; BSIP 3935: 6; BSIP 4313: 5 - Whitmore et al. BSIP 2846: 6; BSIP 2961: 6; BSIP 3700:

7; BSIP 3734: 7; BSIP 5506: 6; FRI 3001: 6 - Wiakabu \& Fani LAE 73308: 3 - Wiakabu \& Yefle LAE 70319: 6 - William 25: 6; 706: 5 - William \& Noor 175: 6 - Womersley NGF 13480: 3; NGF 24781: 3; NGF 41212: 6.

Yuncker 9719: 6.

Zollinger 2427: 8 .

\section{INDEX}

Numbers refer to the species number as used in this paper. The genus name and its synonyms has been referred to page numbers. New names are in bold, accepted names in roman, and synonyms in italics.

\author{
Alstonia ficifolia S. Moore 4 \\ Bleekeria Hassk. [p. 104, 116] \\ ackeringae (Teijsm. \& Binn.) Koidz. 8 \\ coccinea (Teijsm. \& Binn.) Koidz. 9 \\ littoralis (Merr.) Koidz. 8 \\ minima (Markgr.) Merr. \& L.M. Perry 10 \\ salubris (Raf.) Hassk. 6 \\ Calpicarpum G. Don [p. 104, 107] \\ lamarckii G. Don 6 \\ oppositifolium (Lam.) Boiteau 6 \\ Cerbera oppositifolia Lam. 6 \\ platyspermos Gaertn. 6 \\ salutaris Lour. 6 \\ Excavatia Markgr. [p. 104, 116] \\ coccinea (Teijsm. \& Binn.) Markgr. 9 \\ littoralis (Merr.) Markgr. 8 \\ minima Markgr. 10 \\ Kopsia lamarckii B.D. Jacks. 6 \\ Lactaria Rumph. ex Raf. [p. 104, 107] \\ ackeringae Teijsm. \& Binn. 8 \\ acuminata (Trimen ex Valeton) Koidz. 1 \\ calocarpa auct. 8 \\ coccinea Teijsm. \& Binn. 9 \\ glomerata (Blume) Koidz. 5 \\ oppositifolia (Lam.) Kuntze 6 \\ salubris Rumph. ex Raf. 6 \\ Neisosperma Raf. [p. 104, 106] \\ acuminata (Trimen ex Valeton) Fosberg \& \\ Sachet 1 \\ var. acuminata 1 \\ var. apoense (Elmer) Markgr. 2 \\ apoensis (Elmer) Fosberg \& Sachet 2 \\ citrodora (Lauterb.\& K. Schum.) Fosberg \& \\ Sachet 3 \\ ficifolia (S. Moore) Fosberg \& Sachet 4 \\ glomerata (Blume) Fosberg \& Sachet 5 \\ muricata Raf. 6 \\ oppositifolia (Lam.) Fosberg \& Sachet 6 \\ rudis (Markgr.) Fosberg \& Sachet 4 \\ sciadophylla (Markgr.) Fosberg \& Sachet 7
}

Ochrosia Juss. [p. 104]

sect. Echinocaryon F. Muell. [p. 104, 106]

sect. Lactaria F. Muell. [p. 104, 116]

sect. Ochrosia [p. 104, 116]

sect. Phragmochrosia Pichon [p. 104, 107]

subg. Echinocaryon Valeton [p. 104, 106]

subg. Lactaria (F. Muell.) Valeton [p. 104, 116]

ackeringae (Teijsm. \& Binn.) Miq. 8

var. angustifolia Baker f. 8

acuminata Trimen ex Valeton 1

apoensis Elmer 2

basistamina Hendrian 13

borbonica auct. 6

citrodora Lauterb. \& K. Schum. 3

coccinea (Teijsm. \& Binn.) Miq. 9

cowleyi F.M. Bailey 6

elliptica auct. 6

ficifolia (S. Moore) Markgr. 4

glomerata (Blume) F. Muell. 5

littoralis Merr. 8

minima (Markgr.) Fosberg \& Boiteau 10

oppositifolia (Lam.) K. Schum. 6

parviflora sensu Markgr. 6

platyspermos (Gaertn.) A.DC. 6

rudis Markgr. 4

salubris (Raf.) Blume 6

sciadophylla Markgr. 7

syncarpa Markgr. 11

tenimberensis Markgr. 12

Ochrosia sensu Fosberg \& Sachet [p. 104, 116]

Ochrosia sensu Markgr. [p. 107]

Ochrosion platyspermum (Gaertn.) St.-Lag. 6

Paralstonia clusiacea auct. 3

Pseudochrosia Blume [p. 104, 107] glomerata Blume 5 